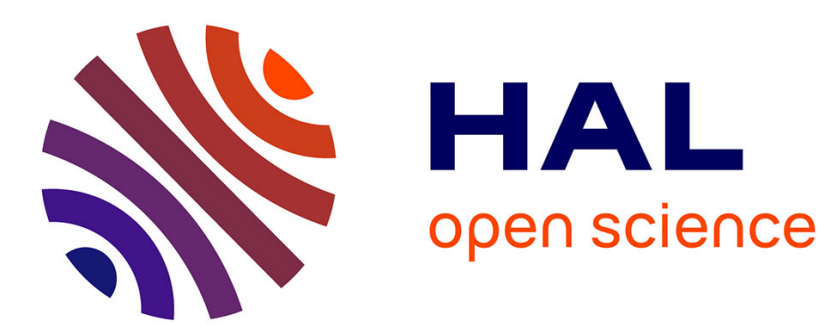

\title{
Tunable multidispersive bands of inductive origin in piezoelectric phononic plates
}

K. Mekrache, R. Sainidou, P. Rembert, N. Stefanou, Bruno Morvan

\section{To cite this version:}

K. Mekrache, R. Sainidou, P. Rembert, N. Stefanou, Bruno Morvan. Tunable multidispersive bands of inductive origin in piezoelectric phononic plates. Journal of Applied Physics, 2021, 130 (19), pp.195106. 10.1063/5.0065184 . hal-03479298

\section{HAL Id: hal-03479298}

https://hal-normandie-univ.archives-ouvertes.fr/hal-03479298

Submitted on 14 Dec 2021

HAL is a multi-disciplinary open access archive for the deposit and dissemination of scientific research documents, whether they are published or not. The documents may come from teaching and research institutions in France or abroad, or from public or private research centers.
L'archive ouverte pluridisciplinaire HAL, est destinée au dépôt et à la diffusion de documents scientifiques de niveau recherche, publiés ou non, émanant des établissements d'enseignement et de recherche français ou étrangers, des laboratoires publics ou privés. 


\title{
Tunable multidispersive bands of inductive origin in piezoelectric phononic plates
}

\author{
K. Mekrache,${ }^{1}$ R. Sainidou, ${ }^{1}$ P. Rembert,,${ }^{1, a)}$ N. Stefanou, ${ }^{2}$ and B. Morvan ${ }^{1}$ \\ 1) Laboratoire Ondes et Milieux Complexes UMR CNRS 6294, UNIHAVRE, Normandie University, 75 rue Bellot, \\ 76600 Le Havre, France \\ ${ }^{2)}$ Section of Condensed Matter Physics, National and Kapodistrian University of Athens, Panepistimioupolis, \\ GR-15784 Athens, Greece
}

(Dated: 18 October 2021)

A variety of multidispersive, localized or extended in frequency, bands, induced by inductance-based external electric circuits in piezoelectric phononic plates, is studied both theoretically and experimentally in this work. Their origin, tightly related to an equivalent $L C$-circuit behavior is analyzed in detail and their interaction with the Lamb-like guided modes of the plate is also discussed. These bands, easily tuned by the choice of the parameters of the external electric circuitry lead to a non-destructive, real-time control of the dispersion characteristics of these structures. Our device and analysis can find application in improvement of surface acoustic wave (SAW) components, by offering additional degrees of freedom.

\section{I. INTRODUCTION}

2 Controlling the dispersion of elastic waves has be3 come a key issue when dealing with sound mitigation, 4 vibration energy harvesting and, more generally, appli5 cations in which elastic waves have to be guided, colli6 mated or trapped. The use of metamaterials has been 7 an important step towards the feasibility of this con8 trol $^{1-3}$ by shaping the dispersion relation of these com9 plex media. Several approaches have been proposed, the 10 most common being the periodic structuration of the 11 medium (phononic crystal) leading to folding of its fre12 quency bands and opening up of band gaps. To tailor the 13 band structure at subwavelength regime, a very promis14 ing means has been the use of the locally-resonant mate15 rials that produce strong dispersive effects related to neg16 ative effective bulk modulus and mass density ${ }^{4-6}$. These 17 structures suffer, however, from an intrinsic narrow fre${ }_{18}$ quency range behavior of the resonant phenomena. To 19 overcome this limitation, a more sophisticated structur20 ing has been recently proposed, facilitated by the use ${ }_{21}$ of new manufacturing techniques, such as 3D printing. 22 The concepts of rainbow metamaterials, gradient index 23 and hierarchical metamaterials allowing to model the dis${ }_{24}$ persion of the waves on a broader frequency range have 25 emerged $^{7-9}$.

26 However all these attractive properties of metamate${ }_{27}$ rials, even in the case of wide-range frequency phenom${ }_{28}$ ena, will stay less appealing, especially for application ${ }_{29}$ purposes, if the question of frequency tunability in their 30 response is not answered. For a fixed geometrical ar31 rangement of the constituents of the complex medium, 32 the resulting frequency response cannot be adapted in 33 real time in a real device. A simple way to introduce 34 this frequency tunability/agility lies in the use of active 35 components whose control can be achieved with the help

a) Electronic mail: pascal.rembert@univ-lehavre.fr
36 of an externally applied field (magnetic ${ }^{10,11}$, electric ${ }^{12}$, ${ }_{37}$ thermal $^{13,14}$, and radiative $\left.{ }^{15,16}\right)$. The mechanical prop38 erties (mass density, elastic moduli) of the active compo39 nent are thus modified, leading to reversible changes in its 40 frequency band structure. Among others, piezoelectric${ }_{41}$ based structures have been shown to be excellent can42 didates for the real-time control of the propagation of 43 elastic waves. The electro-mechanical coupling, natu44 rally present in these materials, offers a versatile tool 45 to shape the effective elastic properties of the metamate46 rial, through a variety of electrically controlled external 47 conditions applied at the structure ${ }^{17-26}$.

48 In this paper, we use a piezoelectric material as a sub49 strate (plate) on which metallic strips (electrodes) are 50 structured. In previous works ${ }^{23-26}$, some of us have 51 shown that propagation of Lamb-like guided modes in 52 these phononic crystal piezoceramic plates exhibits an 53 interesting tunable character easily controlled by exter54 nal electric circuits through appropriate electric bound55 ary conditions (EBCs) applied at the metallic strips. In ${ }_{56}$ particular, the band structure of the plate is significantly 57 modulated by the presence of electric resonant modes 58 originating from the external inductance-loads coupled ${ }_{59}$ to the effective capacitive behavior of the piezoelectric 60 plate. Some basic methodological tools of our analysis ${ }_{61}$ are already presented in Ref. 26. Here, we extend them ${ }_{62}$ in order to give a synthetic panel that these systems can 63 offer. We review under a new light some of the possi64 bilities to mould the dispersion characteristics of tunable ${ }_{65}$ localized bands originating from an equivalent $L C$-circuit ${ }_{66}$ behavior, inherent in piezoelectric phononic plates, when 67 loaded with inductance external circuits. We focus on ${ }_{68}$ three typical representative cases including external $L$ 69 based loads that generate, each of them, a variety of 70 multidispersive, tunable bands of electromagnetic (EM) 71 origin, easily controlled via the external circuitry param72 eters (the inductance, in our case). These cases, first ${ }_{73}$ given in a qualitative schematic manner, cover, respec74 tively, low, intermediate and higher frequency regions in 75 a typical dispersion plot. Moreover, compared to previ- 
${ }_{76}$ ous works ${ }^{23-26}$, an important improvement of our exper- ${ }_{128}$ propriate EBCs at each pair of (up- and down-side) elec77 imental device - especially its electronic part - is made, ${ }_{22}$ trodes. The latter is connected in the most general case 78 towards a wider range of choices in real-time control and ${ }_{130}$ to external electric circuits of characteristic impedance 79 automatization, combined to a robust and promising sig- ${ }_{131} Z^{\mathrm{u}}$ and $Z^{\mathrm{d}}$, as schematically depicted in Fig. 1(c). In this 80 nal processing technique leading to a finer analysis of the ${ }_{132}$ manner, the elementary blocks of length $a_{0}=w+s$ may 81 experimental data. Usually, surface acoustic wave (SAW) 133 have identical $\left(a=a_{0}\right)$ or different $\left(a=\kappa a_{0}, \kappa=2,3, \ldots\right)$ 82 devices offer only input and output ports, restricting the ${ }_{134}$ EBCs throughout the structure, thus tuning at will the 83 analysis exclusively in the frequency domain. Our de- ${ }_{135}$ real periodicity of the crystal. The 1D Brillouin zone 84 vice, allows to probe the surface waves along their path, 136 (BZ) of the crystal is accordingly shortened; we will de85 thus offering the wavevector projection along this path ${ }_{137}$ note its center $\left(k_{1} a / \pi=0\right)$ by $\Gamma$ and its edge $\left(k_{1} a / \pi=1\right)$ 86 as an additional degrees of freedom, which could provide 138 by $\mathrm{X}$.

87 an improvement in the domain of SAW components.

${ }_{88}$ The paper is organized as follows. In Sec. II we present 89 the general characteristics of the materials and structures 90 considered in this study, and provide all necessary details ${ }_{91}$ concerning the experimental setup employed for our mea92 surements, as well as the methods and techniques used in 93 the analysis of the experimental data and in the numer94 ical calculations, that follow in Sec. III. For the different 95 structures under study, the analysis of their dispersion 96 properties, strongly influenced by the external loads, in 97 terms of underlying physical mechanisms responsible for 98 these effects, as well as in comparison with their experi99 mental observation, constitute the main part of Sec. III 100 before concluding the paper in Sec. IV.

\section{II. MATERIALS AND METHODS}

\section{${ }_{39}$ B. Experimental setup}

140 Our experimental setup, though simple, is designed in 141 such a general manner to facilitate modification at will of 142 the EBCs applied at each electrode by connecting exter143 nal circuits (resistive, capacitive, and/or inductive). The 144 current version has substantially improved, as compared 145 to the one used in Ref. 26. First, the plate is inserted into 146 a 1 mm-pitch PCI Express ${ }^{\circledR}$ edge card reader (stack147 able female connector with 98 contacts), allowing con148 nection of the electrodes of the crystal to different elec149 tric impedances, through controlled switches (FST3125). ${ }_{150}$ All connections are now realized in a secure and robust 151 manner avoiding any noise and parasitic effects arising 152 from soldering. Second, a microcontroller (Teensy 3.6) ${ }_{153}$ is used to actuate these switches, thus making possible a 154 real-time control. Apart from the first pair of electrodes 155 reserved for the excitation of the plate, the remaining 47 156 pairs of electrodes will be used to load the plate with ex157 ternal circuits, as shown in Fig. 2(a). Only this part of 158 the plate (we will refer to it as loaded crystal hereafter)

\section{${ }_{103}$ A. Sample structure} ${ }_{104}$ We shall be concerned, in this study, with thin homo- 159 will be utilized to image the frequency response of the
105 geneous piezoceramic plates whose both surfaces are dec- 160 structure, except otherwise stated.

106 orated with periodic one-dimensional (1D) arrays (lattice ${ }_{161} \quad$ A $200 \mathrm{~V}$-amplitude and $0.1 \mu$ s-width pulse signal is ap${ }_{107}$ constant $a_{0}$ ) of parallel and face-to-face metallic strips of 162 plied to the first pair of electrodes at the one edge of the 108 width $w$ and negligible thickness. A schematic represen- ${ }_{163}$ piezoelectric plate, using a Panametrics 5058PR pulse 109 tation of the structure is given in Fig. 1(a). The plate ${ }_{164}$ generator, to excite guided waves within the plate. The 110 is made of PZT (Navy VI) and in all theoretical calcu- 165 incident beam penetrates into the loaded crystal and is 111 lations presented in this paper it will be considered to 166 reflected back at the other edge. To visualize the fre112 be infinite along $x_{1}$ - and $x_{2}$-directions that coincide with 167 quency response of the crystal, we employ an all-electric 113 the transversely isotropic plane of the piezoceramic ma- 168 experimental technique (more details can be found in 114 terial; the $x_{3}$-axis coincides with the polarization axis of 169 Ref. 26). After excitation of the piezoelectric plate, any 115 the material.

170 deformations producing not negligible thickness varia${ }_{116}$ The sample used in the experiments, is a finite square- 171 tions of the plate will be manifested as electric potential 117 shaped plate (edge length $48 \mathrm{~mm}$ and thickness $h=172$ variations on the electrodes through the electromechani$1181.6 \mathrm{~mm}$ ), as can be seen in Fig. 1(b), on both sides of 173 cal coupling that takes place within the piezoelectric ma119 which silver strips (rectangular electrodes) have been de- 174 terial; we exclusively measure these electric potentials ${ }_{120}$ posited periodically; their thickness is of the order of 175 along the plate. To this end, a second PCI card reader, ${ }_{121} 15 \mu \mathrm{m}$ and will be neglected in what follows. In the 176 identical to the first one used for the loading by exter122 real structure, the lattice period dictated by the metallic 177 nal circuits, is connected to the remaining free edge of ${ }_{123}$ strips (width $w=0.7 \mathrm{~mm}$, separation gap $s=0.3 \mathrm{~mm}$ ) 178 the plate [Fig. 2(b)]. The electric potential is recorded ${ }_{124}$ equals $a_{0}=s+w=1 \mathrm{~mm}$, but as we have already ex- 179 with a 10-bit quantification, at each electrode position, ${ }_{125}$ plained in previous works ${ }^{24-26}$ the true lattice constant 180 on the upper or lower side of the plate, with the help of a 126 of the crystal $a$ can be non-destructively extended to be a 181 digital oscilloscope (LeCroy HRO66ZI WaveRunner), the ${ }_{127}$ multiple of $a_{0}$, controlled through the application of ap- 182 ground of the pulse generator being taken as reference; 
(a)

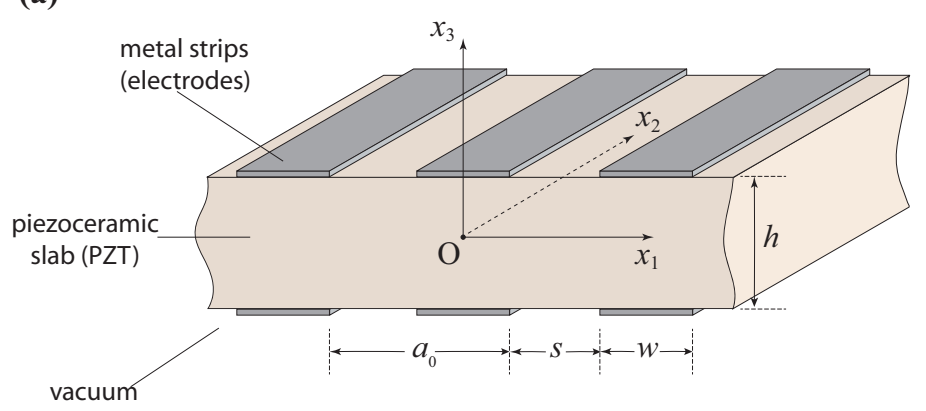

(b)

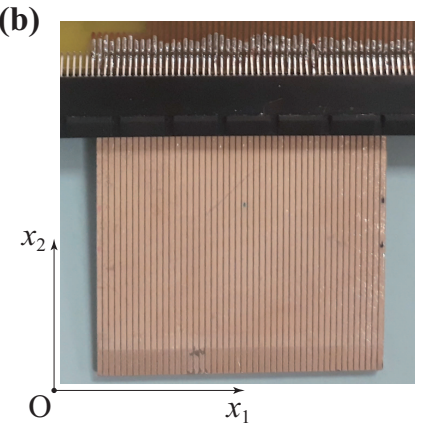

(c)

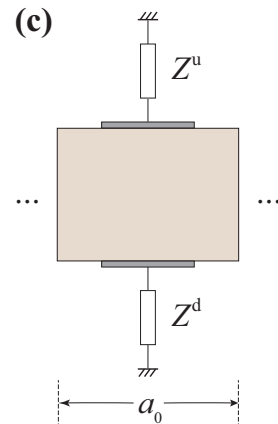

FIG. 1. (a) Schematic representation of the one-dimensional piezoelectric crystal, extended to infinity along $x_{1^{-}}$and $x_{2}$ directions coinciding with the transversely isotropic plane of the piezoceramic material, poled across its thickness along the $x_{3}$ symmetry axis. (b) An image of the fabricated sample with finite dimensions $(48 \mathrm{~mm} \times 48 \mathrm{~mm} \times 1.6 \mathrm{~mm})$, used in the experiments; the metallic strips (width $w=0.7 \mathrm{~mm}$ and separation gap $s=0.3 \mathrm{~mm}$ ) aligned along $x_{2}$-direction are connected at the top edge of the plate to a PCI reader. The structure consists of $N=48$ elementary blocks of length $a_{0}=1$ mm. In (c) an elementary block of the structure loaded with electric circuits of impedance $Z^{\mathrm{u}}$ (upper plate side) and $Z^{\mathrm{d}}$ (lower plate side).

TABLE I. Material parameters for PZT (Navy VI), used in the calculations.

\begin{tabular}{lcc}
\hline the calculations. & Symbol & Value $^{\mathrm{a}}$ \\
\hline \hline Material Property & $c_{11}^{E}$ & 114.0 \\
& $c_{12}^{E}$ & 75.7 \\
Elastic coefficients & $c_{13}^{E}$ & $70.0(72.4)$ \\
$c_{p q}^{E}[\mathrm{GPa}]$ & $c_{33}^{E}$ & $107.0(111.0)$ \\
& $c_{44}^{E}$ & $20.0(26.3)$ \\
& $c_{66}^{E} \mathrm{~b}$ & 19.2 \\
\hline \multirow{2}{*}{ Piezoelectric coefficients } & $e_{15}$ & $14.2(16.2)$ \\
$e_{i p}\left[\mathrm{C} \mathrm{m}^{-2}\right]$ & $e_{31}$ & -2.92 \\
& $e_{33}$ & $20.3(23.4)$ \\
\hline Relative permittivity & $\epsilon_{11}^{S}$ & 2120. \\
coefficients $\epsilon_{p q}^{S}$ & $\epsilon_{33}^{S}$ & 1980. \\
\hline Mass density $\left[\mathrm{kg} \mathrm{m}^{-3}\right]$ & $\rho$ & $7510 .{ }^{\mathrm{c}}(7780)$. \\
\hline \hline
\end{tabular}

a In the parenthesis, we give - if different - the initial values as provided by the manufacturer 28 .

b $c_{66}^{E}=\frac{1}{2}\left(c_{11}^{E}-c_{12}^{E}\right)$.

c Measured.

183 the time window chosen is typically set to $250 \mu \mathrm{s}$, with a ${ }_{184}$ sampling period equal to $5 \mathrm{~ns}$, allowing to observe a few ${ }_{185}$ forward and backward traveling waves after reflections 186 at the edges of the plate. Each measured signal is then 187 averaged 30 times to improve the signal-to-noise ratio. ${ }_{188}$ The above settings ensure a sufficiently high Nyquist fre189 quency and accurate resolution of the spectra obtained 190 through fast Fourier transforms (FFT).

\section{${ }_{191}$ C. Simulations and signal processing}

192 All band structure calculations shown in this paper 193 were obtained by the use of a finite-element commercial ${ }_{232}$ The floating-potential EBCs were used as a bench${ }_{194}$ package $^{27}$ (except otherwise stated). Assuming trans- 233 mark; we are, however, interested in more complex unit

${ }_{195}$ lational invariance along $x_{2}$-direction, the unit cell is ${ }_{234}$ cells. For the systems under study, the dispersion plot
196 reduced to a rectangle and the metallic strips to lines ${ }_{197}$ of zero thickness, able to host surface electric charges. ${ }_{198}$ The classical constitutive equations of piezoelectricity 199 (stresses as functions of strains and electric field) apply 200 at the surface of the rectangle. The boundary condi201 tions are: (i) continuity of all fields between adjacent ${ }_{202}$ cells and periodic (Bloch) conditions at the left and right 203 boundaries of the same cell, and (ii) at the upper and 204 lower edges of the cell, free stress conditions, together 205 with discontinuity of the normal component of the elec206 tric displacement field related to the surface charge den207 sity across the interface, assuming a uniform distribution 208 of charge along the line representing the metallic strip. 209 Under this last assumption, the whole electrode is seen 210 as an electric node, where Ohm's relation involving ex211 ternal impedance loads can be applied. We recall that ${ }_{212}$ a full comparison between an ab-initio analytical model ${ }_{213}$ using the above assumptions and this FEM approach was 214 given in Ref. 25, leading to practically identical (graphi215 cally indiscernible) results for the band diagrams of these 216 systems. We note in passing that only the positive part of 217 the BZ will be represented, since the dispersion diagrams ${ }_{218}$ are symmetric with respect to the vertical (frequency) 219 axis, in all cases discussed here. The material param220 eters (elastic, piezoelectric and coupling constants) for ${ }_{221}$ PZT used in the simulations are shown in Table I and 222 were determined by adjusting the dispersion relation of 223 a floating-potential crystal to the corresponding experi224 mental data. The experimental dispersion relation $\omega\left(k_{1}\right)$, ${ }_{225} \omega=2 \pi f$ being the angular frequency, was obtained, af226 ter applying a 2D FFT of the potential signals $V\left(x_{1, n}, t\right)$ 227 measured at each electrode position $x_{1, n}, n=2,3, \ldots, 48$, 228 of the upper and lower side of the corresponding finite 229 plate, following the procedure described in Ref. 26. The 230 simulation results agree very well with the experimental data (tolerance better than $3.2 \%$ ). 
(a)

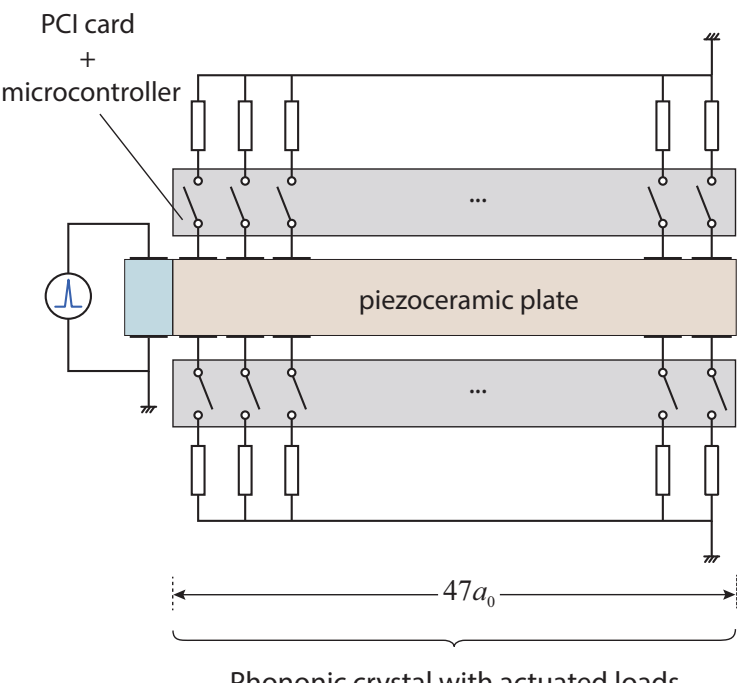

(b)

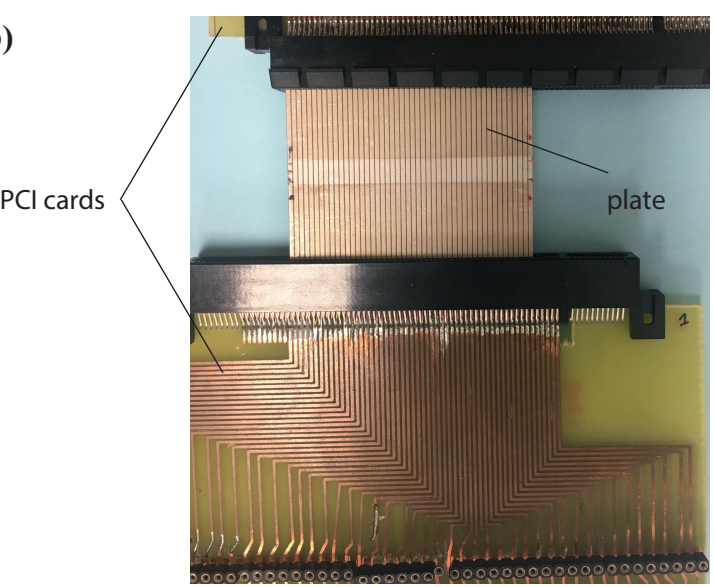

FIG. 2. The experimental setup used for the study of the phononic plate of Fig. 1: (a) schematic representation, and, (b) a view of the device. Excitation takes place at the left end of the plate and loading by external circuits at its remaining part through controlled EBCs.

235 is more crowded: several eigenmode trajectories coexist 236 in the $\omega-k_{1}$ space at neighboring points and cannot be ${ }_{237}$ easily resolved, after a 2D FFT is performed. To better 238 resolve these separate contributions, we opt for a different 239 method of analysis, by adapting an autoregressive model 240 to two dimensions (AR2D), i.e., to both space and time 241 directions ${ }^{29}$. The results obtained in that manner agree 242 with the corresponding picture obtained by a 2D FFT, 243 without suffering from a wide $\omega-k_{1}$ peak-character.

\section{III. RESULTS AND DISCUSSION}

245

246 In what follows, we will focus on three configurations, 247 all of them using inductance loads on the unit cell of the 200 ${ }_{248}$ crystal, shown schematically in the left panel of Fig. 3; 261 flat and one infinitely extended hyperbola-like frequency (a)
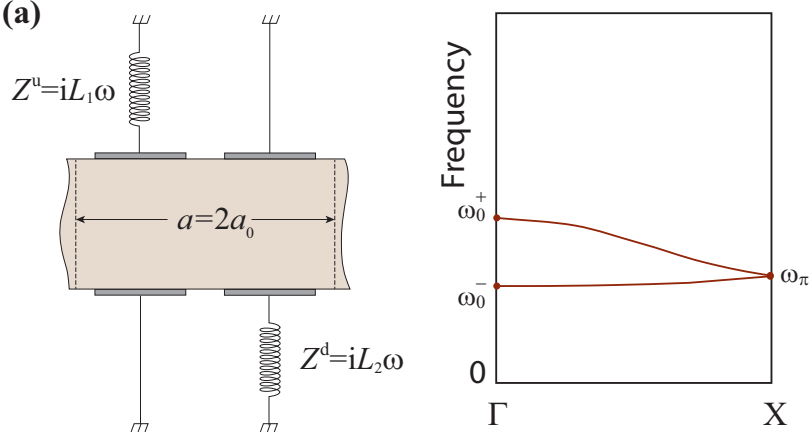

(b)

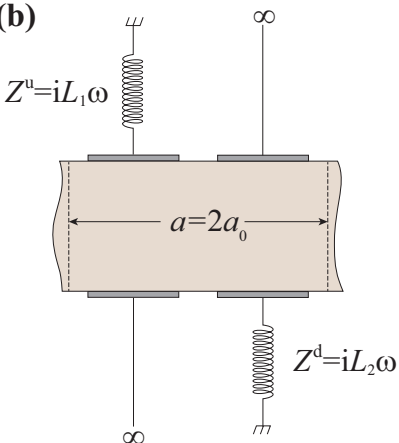

(c)
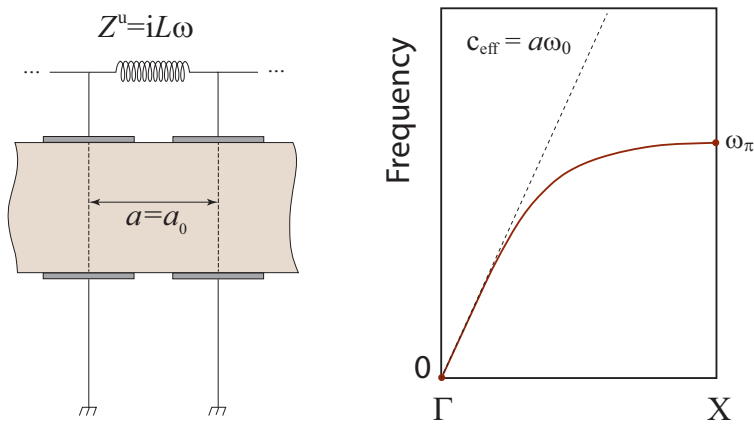

FIG. 3. The three configurations with inductive loads under study. Left panel: the unit cell of each crystal with different electric boundary conditions applied at the metallic strips [(a) grounded-inductance two-atom crystal, (b) floating-potentialinductance two-atom crystal, and (c) intercellular adjacent inductance-loaded crystal]. Right panel: schematic representation of the corresponding dispersion relation of the induced $L C$-origin bands (for $L_{1}=L_{2}$, when applicable).

249 their interest lies on the unusual multidispersive form 250 of localized and/or extended frequency bands of induc251 tive origin that this circuitry may offer. More precisely, 252 the first two cases correspond to two-atom unit cells $253\left(a=2 a_{0}\right)$, constructed by combination of two antipar254 allel elementary blocks (of width $a_{0}$ ), each of them hav255 ing a $L$-load on the one side of the plate, the other-side 256 electrode being either shunted [Fig. 3(a)] or in floating 257 potential [Fig. 3(b)]. Though inspired from Ref. 26, the 258 resulting structures offer new possibilities, since they pos259 sess inversion symmetry and exhibit, respectively, a set

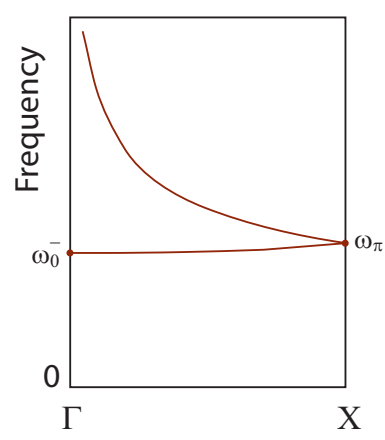


262 bands, as schematically depicted in the right panel of ${ }_{263}$ Fig. 3. A third configuration [see Fig. 3(c)] consists 264 of a $L$-load connecting adjacent strips, the EBCs con${ }_{265}$ serve, in that case, the spatial periodicity of the metallic 266 array $\left(a=a_{0}\right)$. The corresponding modes originating 267 from this circuitry exhibit a linear dispersion behavior ${ }_{268}$ with an effective medium slope $c_{\text {eff }}=a \omega_{0}$ at the long269 wavelength limit which bends and tends to a zero-group270 velocity branch at $\mathrm{X}$ point. It is worth noting that such 271 a configuration is a direct analogue of the unit cell of 272 a transmission line governed by the well-known telegra273 pher's equation.

${ }_{274}$ The different characteristic angular frequencies $\omega_{0}^{ \pm}, \omega_{0}$, ${ }_{275} \omega_{\pi}$, appearing in Fig. 3 to describe qualitatively the dis276 persion of these eigenmodes at $\Gamma$ and $X$ points of the ${ }_{277} \mathrm{BZ}$, depend of course of the choice of $L$ and of some ${ }_{278}$ characteristic capacitance values $C, C_{s}, C_{1}$, and $C_{2}$ that 279 describe effectively the intrinsic behavior of the piezoelec280 tric plate in terms of equivalent circuits. More details are 281 given in Appendix A. We note that this analysis for the 282 two-atom crystals reveals higher-order interactions of ca${ }_{283}$ pacitive origin within the plate which takes place between 284 neighbors of adjacent cells and opposite sides, compared 285 to previous demonstrations ${ }^{26}$.

\section{A. Grounded and inductance loaded two-atom unit cell}

${ }_{287}$ To begin with we shall examine the case of up- and 288 down-side inductance loads alternated with grounded 289 strips. The unit cell is thus a two-atom molecule ana$290 \log$ with inversion symmetry if $L_{1}=L_{2}$, or with iden291 tity operation symmetry only, if $L_{1} \neq L_{2}$, as shown in ${ }_{292}$ Fig. 3(a). Aiming to analyze its frequency band structure 293 both theoretically and experimentally, we find it useful to 294 focus first on the frequency response of the correspond295 ing monatomic crystal of lattice constant $a=a_{0}$ [sup296 pose only the left half part of the unit cell of Fig. 3(a)]. ${ }_{297}$ In the absence of piezoelectric coupling $\left(e_{i j}=0\right)$, the 317 becomes shorter $\left(a=2 a_{0}\right)$. An additional folding at 298 eigenmodes of the crystal are separated into two indepen- $318 k_{1} a_{0} / \pi=0.5$ of the dispersion plot is dictated and, as a 299 dent, non-interacting subspaces: the elastic-displacement 319 result, the EM resonant band is thus split into two sepa300 Lamb guided modes of the plate (labelled, hereafter, with 320 rate flat minibands, a more dispersive, $\omega^{+}\left(k_{1}\right)$, extending $301 \mathrm{~A}$ for antisymmetric and $\mathrm{S}$ for symmetric modes, follow- 321 from $0.839 \mathrm{MHz}\left(k_{1}=0\right)$ to $0.732 \mathrm{MHz}\left(k_{1}=\pi / a\right)$, and a 302 ing standard notation used for Lamb modes in plates), 322 less dispersive, $\omega^{-}\left(k_{1}\right)$, extending from $0.708 \mathrm{MHz}\left(k_{1}=\right.$ 303 simply folded at the BZ edge $\left(k_{1} a_{0} / \pi=1\right)$ and the EM 3230$)$ to $0.732 \mathrm{MHz}\left(k_{1}=\pi / a\right)$. These bands compare very 304 modes which originate from an $L C$-equivalent circuit be- 324 well (relative error better than $\pm 0.9 \%$ ) to a tight-binding 305 havior of the unit cell. The electric resonances of these 325 description ${ }^{30}$ with first-neighbour interactions between 306 individual $L C$ atoms interact between each other and 326 atomic sites [blue dashed lines in Fig. 4(a)] that leads ${ }_{307}$ form a cos-like resonant band. In Fig. 4(a) we show the 327 to $\omega^{ \pm}\left(k_{1}\right)=\left[\omega_{\text {at }}^{2}+2 \gamma_{1} \cos k_{1} a \pm 2 \gamma_{2} \cos \left(k_{1} a / 2\right)\right]^{1 / 2}, \gamma_{1}=$ 308 calculated - using a finite-element method - frequency $328 \gamma(a), \gamma_{2}=\gamma(R)$ being the overlap integrals $\gamma$ depending 309 band structure of such a crystal, the first subspace shown 329 on the distance $a=2 a_{0}$ and $R=\sqrt{a_{0}^{2}+h^{2}}=1.89 a_{0}$ 310 in gray lines, the second one in blue dotted line, extend- 330 between the centers of the atomic potential functions 311 ing from $1.294 \mathrm{MHz}\left(k_{1}=0\right)$ to $0.597 \mathrm{MHz}\left(k_{1}=\pi / a_{0}\right)$. 331 and $\omega_{\text {at }}$ the eigenfrequency of an isolated atom. By a ${ }_{312}$ We note in passing that this zero coupling analysis, al- 332 best fitting procedure we find $\omega_{\text {at }} /(2 \pi)=0.753 \mathrm{MHz}$, 313 ready presented in previous works ${ }^{24,26}$, can be employed $333 \sqrt{\gamma_{1}} /(2 \pi)=0.128 \mathrm{MHz}$, and $\sqrt{\gamma_{2}} /(2 \pi)=0.214 \mathrm{MHz}$. 314 to visualize the bands of EM origin before hybridization. 334 The $\omega^{ \pm}\left(k_{1}\right)$ bands are independently reproduced by the 315 When two antiparallel atoms are brought together to 335 electric-circuit picture [see Eq. (A1) in Appendix A 1, 316 form a two-atom unit cell as shown in Fig. $3(\mathrm{a})$ the BZ 336 with $C=0.103 \mathrm{nF}, C_{s}=0.552 \mathrm{nF}, C_{1}=0.0348 \mathrm{nF}$, and 
${ }_{337} C_{2}=0.116 \mathrm{nF}$ obtained by a best fitting procedure], also 338 in accordance with the above tight-binding model.

339 A more careful look at the potential field functions at $\Gamma$ 340 point plotted within the unit cell (see top panel of Fig. 4) 341 reveals a bonding $\left(\Phi_{+}=\mathcal{C}_{+}\left(\phi_{1}+\phi_{2}\right)\right)$ and antibonding ${ }_{342}\left(\Phi_{-}=\mathcal{C}_{-}\left(\phi_{1}-\phi_{2}\right)\right)$ character in analogy with a diatomic ${ }_{343}$ molecule picture in quantum physics, $\phi_{1}$ and $\phi_{2}$ being the 344 two atomic potential functions and $\mathcal{C}_{ \pm}$appropriate coef345 ficients. Along $\Gamma \mathrm{X}$ direction the potential fields evolve 346 progressively towards a more weighted one-atom picture 347 with a double-degenerate state at X point (see two right ${ }_{348}$ plots of the top panel in Fig. 4), this degeneracy being 349 lifted when inversion symmetry disappears $\left(L_{1} \neq L_{2}\right)$.

350 This picture is further modified when piezoelectric cou351 pling is taken into account $\left(e_{i j} \neq 0\right)$, as it should be in 352 a real system. The result of our calculation is shown in ${ }_{353}$ Fig. 4(b). As a first remark, one observes a blue shift 354 for the Lamb guided modes and a red shift for the EM 355 origin resonant bands. And more importantly, the two 356 eigenmode subspaces interact between each other and, in 357 general, one expects the formation of avoided crossings 358 between modes of the same symmetry. The lower in fre359 quency EM resonant band $\omega^{-}\left(k_{1}\right)$ interacts with both the ${ }_{360} S_{0}$ branch and the first folded $A_{0}$ branch, and similarly 361 the higher in frequency EM band $\omega^{+}\left(k_{1}\right)$ interacts with 362 both the $A_{1}$ branch and the first folded $S_{0}$ branch, thus 363 leading to narrow selective absolute gaps (yellow-shaded 364 regions). A slight detuning between $L_{1}$ and $L_{2}$ lowers 365 further the symmetry of the system (inversion is now in366 valid) thus resulting in interactions, though very weak, 367 between all bands [see Fig. 4(c)].

${ }_{368}$ Next, we confirm the above predictions, by their exper369 imental verification. We measured the potential signal 370 $V\left(x_{1, n}, t\right)$ along the $n=2,3, \ldots, 48$ electrode positions 371 located at the upper side of the plate, and after appli372 cation of the AR2D model, we obtain the experimental ${ }_{373} V\left(\omega, k_{1}\right)$, whose amplitude is shown in Fig. 5(a), and 374 close to it, in Fig. 5(b), the calculated frequency band 375 structure shown in Fig. 4(b). The different branches are 376 color-indexed with the value of the potential, averaged on
377 the area of the upper-side electrodes: the darker the traз78 jectory, the weaker the electromechanical coupling, that 379 takes place within the plate, is.

${ }_{380}$ The experimental dispersion plot mainly reveals the 382 of the $1^{\text {st }}$ and negative part of the $2^{\text {nd }}$ BZ, that com383 pare well to the corresponding theoretical curve with ef384 fective medium slope of $3013 \mathrm{~m} \mathrm{~s}^{-1}$ at the long wave385 length limit. In addition, one observes some bright spots 386 spanning the frequency region between 0.6 and $0.7 \mathrm{MHz}$, 387 that could correspond to the localized modes of EM 388 origin in Fig. $5(\mathrm{~b})$ at about $0.58 \mathrm{MHz}$ for $\omega^{-}\left(k_{1}\right)$ and 389 at about $0.67 \mathrm{MHz}$ for $\omega^{+}\left(k_{1}\right)$. At higher frequencies 390 but below $1 \mathrm{MHz}$, two more trajectories are experimen391 tally observed, belonging probably to $A_{1}$-like and $S_{0}$-like 392 branches, visible in the positive part of the $1^{\text {st }}$ and $2^{\text {nd }}$ ${ }_{393} \mathrm{BZ}$, respectively (but also in the negative part of the $2^{\text {nd }}$ 394 and $1^{\text {st }}$ BZ). The comparison between theory and experi-

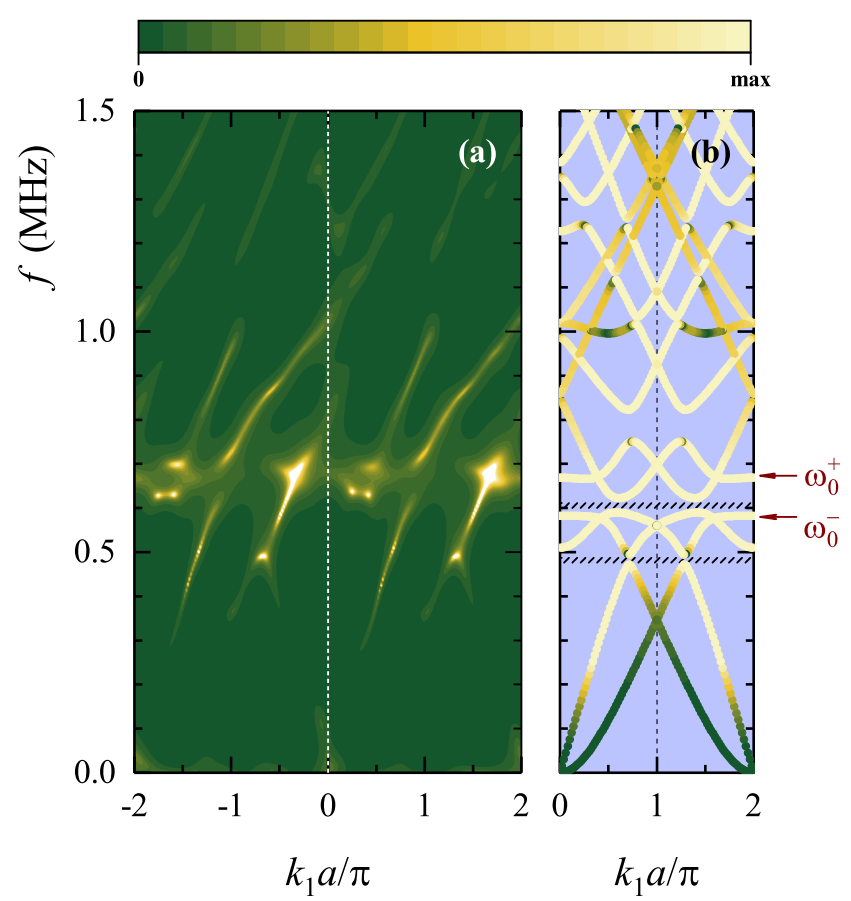

FIG. 5. Frequency band structure of the diatomic grounded$L$ piezoelectric phononic crystal (lattice constant $a=2 a_{0}=$ $2 \mathrm{~mm}$ ) with EBCs depicted in Fig. 3(a). (a) Experimental dispersion plot for the finite crystal, consisting of $N=24$ unit cells, obtained by use of the AR2D model on the electricpotential signal recorded at the upper-side electrode positions. (b) Calculated dispersion plot obtained through finite-element numerical simulations for the corresponding infinite crystal for $L=30 \mu \mathrm{H}$. In (a) the color map is saturated; in (b) the trajectories are color-indexed with the average potential on the upper-side electrodes of the unit cell. Red arrows and hatched areas denote, respectively, frequency bounds of the EM bands at $\Gamma$ point and absolute gaps.

395 ment is, however, not straightforward, especially because 396 the finite-element simulations predict much more active 397 branches than seen experimentally.

\section{B. Floating potential and inductance loaded two-atom 399 unit cell}

If we replace the two grounded ends of the previous 401 structure by free floating potential (FP) ends, we obtain 402 the unit cell shown in Fig. 3(b). A similar methodol403 ogy can be developed as that given in Sec. III A in order 404 to follow the formation of the resonant bands; the main 405 difference lies in the specific form of this band before hy406 bridization. As it can be easily shown ${ }^{31}$ this branch is 407 hyperbolic for the corresponding monatomic crystal [con${ }^{408}$ sider only the left half part of the unit cell of Fig. 3(b)] 409 and when constructing the diatomic unit cell, the fold410 ing results in two branches, one hyperbola-like, $\omega^{+}\left(k_{1}\right)$, ${ }_{411}$ extended in frequency, the other, $\omega^{-}\left(k_{1}\right)$, relatively flat. ${ }_{412}$ When $L_{1}=L_{2}$ these two distinct bands degenerate to 


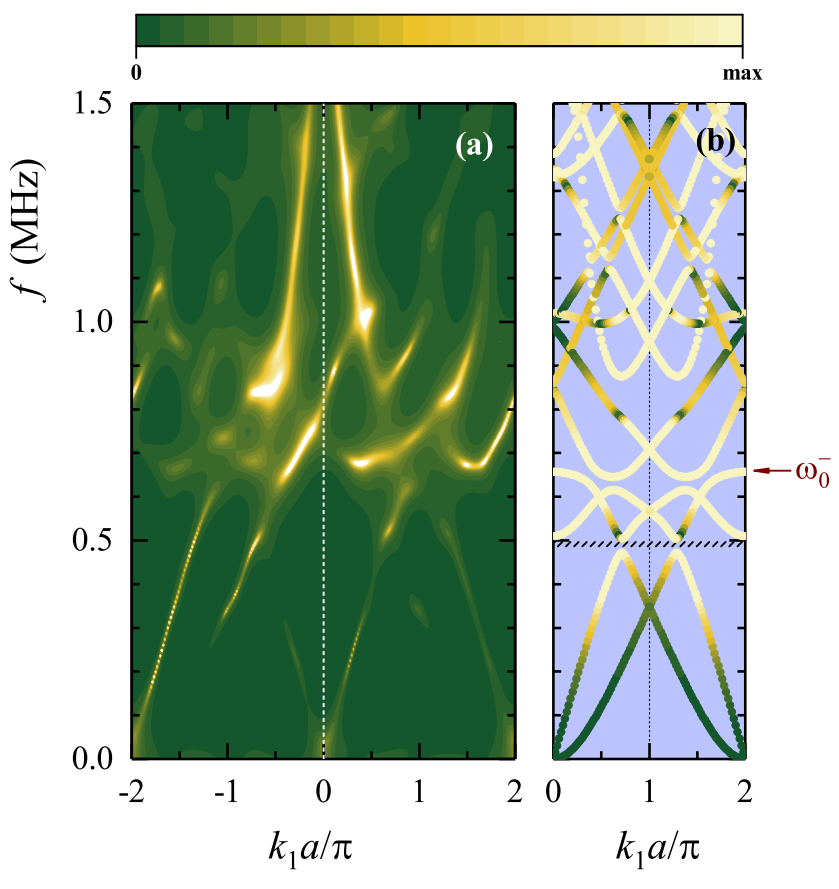

FIG. 6. Frequency band structure of the floating-potential$L$ piezoelectric phononic crystal (lattice constant $a=2 a_{0}=$ $2 \mathrm{~mm}$ ) with EBCs depicted in Fig. 3(b). (a) Experimental dispersion plot for the finite crystal, consisting of $N=24$ unit cells, obtained by use of the AR2D model on the electricpotential signal recorded at the upper-side electrode positions. (b) Calculated dispersion plot obtained through finite-element numerical simulations for the corresponding infinite crystal for $L=30 \mu \mathrm{H}$. In (a) the color map is saturated; in (b) the trajectories are color-indexed with the average potential on the upper-side electrodes of the unit cell. Red arrow and hatched area denote, respectively, frequency bounds of the EM band at $\Gamma$ point and absolute gap.

${ }_{413}$ a common frequency $\omega_{\pi}$ at $\mathrm{X}$ point; again this degener414 acy originating from the inversion symmetry of the unit 415 cell, is lifted if $L_{1} \neq L_{2}$. The finite frequency point $416 \omega_{0}^{-}=\omega^{-}\left(k_{1}=0\right)$ can be tuned through the choice of $L$. ${ }_{417}$ Analytic expressions are derived in terms of an equiva${ }_{418}$ lent periodic transmission-line picture, as detailed in Ap${ }_{419}$ pendix A 2.

${ }_{420}$ The experimental frequency band structure of our ${ }_{421}$ finite-size sample is obtained following the same pro${ }_{422}$ cedure, as for the previous case of grounded crystal ${ }_{423}$ (Sec. III A). The results are shown in Fig. 6(a), and next ${ }_{424}$ to it [Fig. 6(b)] the calculated frequency band structure ${ }_{425}$ for the corresponding infinite crystal, assuming again a ${ }_{426}$ pure $L$-component $(L=30 \mu \mathrm{H})$. In the experimental ${ }_{427}$ picture, three frequency regions are distinguished: be${ }_{428}$ low $0.6 \mathrm{MHz}$ the $S_{0}$-like and $A_{0}$-like branches are clearly ${ }_{429}$ identified, in good agreement with those predicted the430 oretically, and this was also the case for the grounded 431 crystal (Fig. 5). Next, for frequencies above $0.6 \mathrm{MHz}$ 432 and below 1.0 MHz some bright spots are observed but a ${ }_{433}$ one-to-one comparison to the theoretical picture becomes
${ }_{434}$ not straightforward. Finally, above $1.0 \mathrm{MHz}$ the two ex435 tended, hyperbolic branches become dominant and are ${ }_{436}$ clearly observed within the $1^{\text {st }} \mathrm{BZ}$, in both its positive ${ }_{437}$ and negative part.

${ }_{438}$ In both cases examined up to now, we remark that the 439 experimental dispersion plots [Figs. 5(a) and 6(a)] do not 440 reveal all branches predicted theoretically, and, more im${ }_{441}$ portantly, symmetry operations such as translation sym${ }_{442}$ metry by a reciprocal lattice vector, $\omega\left(k_{1}\right)=\omega\left(k_{1}+\frac{2 \pi}{a}\right)$, 443 and/or mirror symmetry, $\omega\left(k_{1}\right)=\omega\left(-k_{1}\right)$, are not ob444 served for all trajectories. This could be explained by ${ }_{445}$ finite size effects and excitation that favors some of the ${ }_{446}$ branches with respect to others. To facilitate compari${ }_{447}$ son to the theoretical picture, we artificially restore the 448 above said lost symmetries, by taking the average on the 449 positive- $k_{1}$ and negative- $k_{1}$ part after, i) horizontal flip450 ping, and, ii) horizontal translation by 2 (in reduced $k_{1}$ 451 axis units) of the latter. These two operations lead to ${ }_{452}$ a picture with mirror symmetry with respect to vertical ${ }_{453}$ (frequency) axis at both $\Gamma$ and $\mathrm{X}$ points, thus the $\Gamma \mathrm{X}$ ${ }_{454}$ direction is sufficient for any analysis. The results are ${ }_{455}$ shown in Fig. 7, for the grounded crystal [left panel, plot ${ }_{456}$ (a)] and for the floating-potential crystal [right panel, ${ }_{457}$ plot (c)]. Their comparison to the corresponding theo${ }_{458}$ retical predictions [Figs. 5(b) and 6(b)] reveals a red shift 459 of the simulated EM modes. Obviously, the reality, far 460 away from the numerous simplifications assumed in the 461 simulations, includes finite size effects, different elastic 462 boundary conditions at the edges of the plate due to the 463 use of the PCI cards, and, real-electronics effects, present 464 in the circuitry, that were neglected in the previous analy465 sis. In fact, a $Z_{e}$ impedance load (see Appendix B) will be ${ }_{466}$ considered instead of the idealized $Z^{\mathrm{u}}=Z^{\mathrm{d}}=Z_{L}=\mathrm{i} L \omega$ ${ }_{467}$ generated by a pure $L$-component. In plots (b) and (d) ${ }_{468}$ of Fig. 7 we show the calculated band structures that 469 correspond to those of Figs. 5(b) and 6(b), but for the ${ }_{470}$ real load $Z_{e}[\mathrm{Eq} .(\mathrm{B} 1)]$ instead of the pure inductive load ${ }_{471}$ with $L=30 \mu \mathrm{H}$. The use of $Z_{e}$ blue-shifts the EM ${ }_{472}$ modes [by about $\sim 10 \%$ for $\omega_{0}^{ \pm}$with respect to those 473 of Figs. 5(b) and 6(b), indicated here by red arrows as 474 guides to the eye] and improves the comparison between 475 experimental data and theoretical simulations. The EM 476 modes manifest themselves as two flat bands lying in the 477 frequency range from 0.6 to $0.75 \mathrm{MHz}$ for the grounded${ }_{478} L$ crystal [Fig. $7(\mathrm{~b})$ ] in rather good agreement with the 479 experimental picture [bright spots extending from 0.63 480 to $0.71 \mathrm{MHz}$ in Fig. 7(a)]. For the floating-potential- $L$ ${ }_{481}$ crystal [Fig. $7(d)$ ] the EM modes manifest themselves, on ${ }_{482}$ the one hand, as a flat band centered at $0.7 \mathrm{MHz}$ and, on 483 the other hand, as a hyperbolic branch which interacts ${ }_{484}$ strongly with the $A_{1}$-like branch and spans the whole 485 frequency range above $0.9 \mathrm{MHz}$; these compare well to 486 the experimental picture [Fig. 7(c)] that reveals, respec${ }_{487}$ tively, a flat bright segment from 0.66 to $0.75 \mathrm{MHz}$ and 488 a hyperbolic branch above $0.82 \mathrm{MHz}$.

We note in passing the appearance of narrow, selective, 40 absolute frequency gaps for both systems [see hatched ar491 eas in Figs. 7(b), (d)] originating from avoided-crossing 


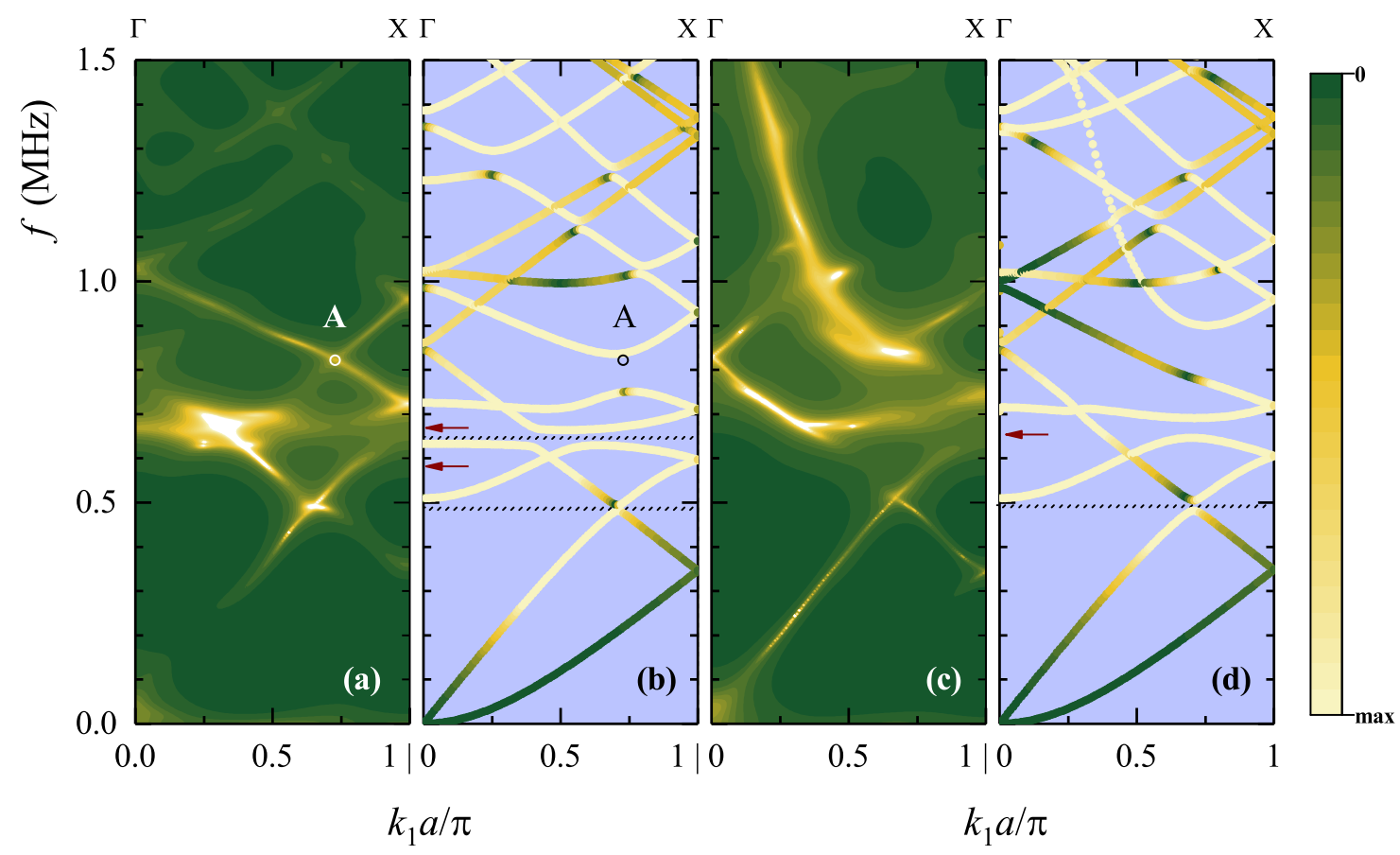

FIG. 7. Experimental frequency band structure of the diatomic (a) grounded- $L$ and (c) floating-potential- $L$ piezoelectric phononic crystals, after reduction along $\Gamma X$ direction (see text) of their initial map plots, shown respectively in Figs. $5(\mathrm{a})$ and 6(a). In (b) and (d) the corresponding calculated dispersion plots for the infinite crystals, assuming real loads $Z_{e}$ instead of the pure inductive ones $Z_{\mathrm{L}}(L=30 \mu \mathrm{H})$. Red arrows denote the frequency positions of the EM bands at $\Gamma$ point as predicted for the case of $Z_{\mathrm{L}}$ instead of $Z_{e}$ [see Figs. 5(b) and 6(b), respectively]; hatched areas indicate, as usually, absolute gaps.

492 between bands of the same symmetry. Therefore, one ex- 520 reason could be the modification of this individual-atom ${ }_{493}$ pects that even structures with a small number of units 521 load $Z_{e}$ (we recall that its identification has been real494 will exhibit such type of gaps ${ }^{32}$. Their frequency po- 522 ized under isolated conditions, outside the crystal) when 495 sition is indirectly controlled through $\omega_{0}$ values scaling 523 it is introduced in our device including the plate itself 496 as $\sim L^{-1 / 2}$ [see Appendix A]. Their width is narrow, 524 and the system of two PCI cards. On the other hand, ${ }_{497}$ but possible enlargement could be achieved with elec- 525 simulations still constitute idealized models, since they 498 tronic components facilitating the degree of interaction 526 neglect any finite size effects and leakage of elastic and 499 [e.g., negative capacitors $\left.{ }^{25}\right]$.

${ }_{527}$ EM modes in the surrounding medium (air). For the 500 A careful global evaluation of the picture obtained by 501 both models used for the simulations, i.e., $Z_{L}$ and $Z_{e}$, ${ }_{502}$ for the two crystals under study, suggests that none of ${ }_{503}$ these can perfectly describe the experimental picture in 504 terms of frequency position of the EM modes (they are in 505 general slightly shifted, but the hyperbolic branch clearly 506 blue-shifted in the simulations) and in terms of degree of 507 interaction that takes place in the avoided-crossing re508 gions when EM and Lamb-like modes cross each other ${ }_{533}$ As a last case, we examine a crystal plate with inter509 [weak avoiding is experimentally observed, while a strong 534 cellular connection of adjacent strips located at the same 510 one is predicted, e.g. at point A in Figs. 7(a), (b)]. ${ }_{535}$ side of the plate (say the upper one) via an inductance 511 Deviations are also observed between some dark branch 536 load $L$, the other-side strips being grounded, as depicted 512 segments (vanishing potential value along them), while ${ }_{537}$ in Fig. 3(c). These EBCs conserve the periodicity of the 513 predicted highlighted in theory, e.g., the $A_{1}$-like branch 538 metallic array. The numerically predicted band struc514 which is theoretically predicted with a cut-off frequency 539 ture of that crystal (lattice constant $a=a_{0}$ ) is shown in 515 close to $0.5 \mathrm{MHz}$ is not experimentally discernible. We ${ }_{540}$ Fig. 8 for two distinct inductance values: $L=150 \mu \mathrm{H}$ 516 deduce that even if a pure $L$-component is too simple ${ }_{541}$ [plot (a)] and $L=30 \mu \mathrm{H}$ [plot (b)]. The most striking ${ }_{517}$ to reproduce exactly the experimental picture of the dis- 542 feature of these dispersion plots is the appearance of a 518 persion plot of these crystals, the use of the experimen- 543 linear-dispersion branch at the long-wavelength limit, in 519 tally identified load $Z_{e}$ is not sufficient either. A possible ${ }_{544}$ addition to the traditionally expected $S_{0}$-like and $A_{0}$-like 

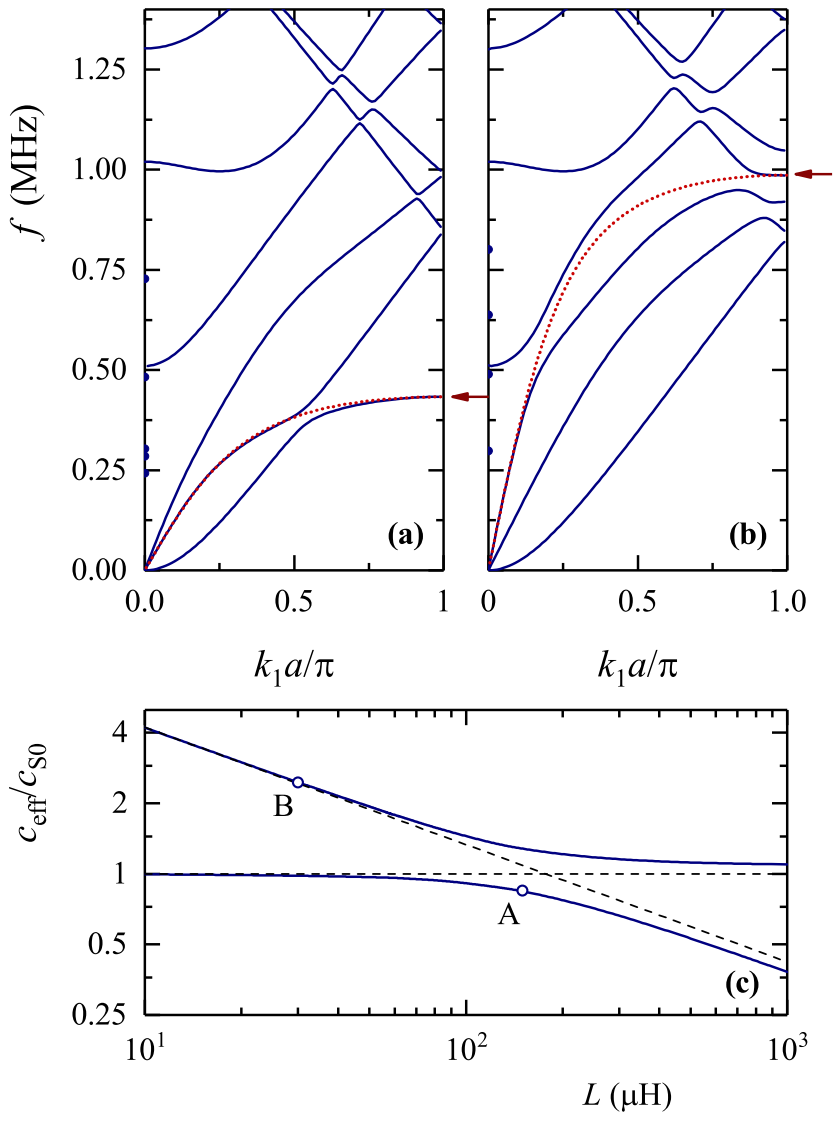

FIG. 8. Calculated frequency band structure for a monoatomic crystal of lattice constant $a=a_{0}$ whose unit cell is depicted in Fig. 3(c) with (a) $L=150 \mu \mathrm{H}$ and (b) $L=30 \mu \mathrm{H}$. The dotted red lines represent an estimate of the unhybridized $L$-induced band, applying Eq. (A3) for appropriate values of the parameters $C, C_{s}$ (see text), with starting parameters $\omega_{\pi} /(2 \pi)$ (indicated by red arrows) and the longwavelength slope, $c_{\mathrm{eff}}$, of this frequency branch. (c) Evolution of the slope of the two linear branches at $\omega \rightarrow 0$ for several values of the inductance load $L$. Horizontal and oblique dashed lines represent, respectively, the effective medium slope of the $S_{0}$ branch of the corresponding grounded crystal and the $L$ induced branch from Eq. (A3) calculated for $C, C_{s}$ determined for $L=10 \mu \mathrm{H}$. Points A and B correspond to the $L$-induced bands (red dotted curves) of plots (a) and (b), respectively.

545 branches emerging at the same frequency range. The gen${ }_{546}$ eral form of that $L$-induced branch when the piezoelectric 547 coupling is switched off $\left(e_{i j}=0\right)$ follows the schematic 548 representation given in the right plot of Fig. 3(c) and ${ }_{549}$ is described by the relation (A3) for some appropriate ${ }_{550}$ values $C, C_{S}$ in an equivalent periodic transmission line ${ }_{551}$ picture. The slope, $c_{\text {eff }}$, of this $L$-branch is tuned via 552 the choice of $L$, and covers a wide range of effective ${ }_{553}$ medium velocities not easily encountered in ordinary ma${ }_{554}$ terials. The equivalent transmission line model presented ${ }_{555}$ in Appendix A predicts a scaling $\sim L^{-1 / 2}$ for given $(L-$ ${ }_{556}$ independent) $C$ and $C_{s}$ values (we find $C=0.645 \mathrm{nF}$ and ${ }_{557} C_{s}=0.593 \mathrm{nF}$, for the crystal under study). When this
558 coupling is switched on $\left(e_{i j} \neq 0\right)$, as it should be in a real 559 system, the $L$-induced band interacts with the same sym560 metry bands of Lamb eigenmodes and generates avoided561 crossing effects. To facilitate its visualization before hy562 bridization, we apply the same relation [Eq. (A3), with 563 different values of $C, C_{s}$, reflecting all coupling effects] to 564 model its unhybridized form, represented in Fig. 8(a) and 565 (b) by red dotted lines: after a careful reading of these 566 dispersion plots, we identify the two starting parameters, ${ }_{567} \omega_{\pi}$ (indicated by a red arrow), and, $c_{\text {eff }}$, the effective568 medium slope of this branch at the long-wavelength limit, ${ }_{569}$ used to deduce the internal model capacitance parame570 ters $\left(C=0.737 \mathrm{nF}\right.$ and $C_{s}=0.868 \mathrm{nF}$, for $L=30 \mu \mathrm{H}$; ${ }_{571} C=1.413 \mathrm{nF}$ and $C_{s}=0.900 \mathrm{nF}$, for $L=150 \mu \mathrm{H}$ ).

572 For the case of $L=150 \mu \mathrm{H}$ [Fig. 8(a)], the $L$ ${ }_{573}$ band with $c_{\text {eff }}=2560 \mathrm{~m} \mathrm{~s}^{-1}$ interacts weakly with the ${ }_{574} A_{0}$-like branch, and a small avoided-crossing occurs at 575 about $0.375 \mathrm{MHz}$; the other linear, $S_{0}$-like, branch has ${ }_{576} c_{\text {eff }}=3850 \mathrm{~m} \mathrm{~s}^{-1}$, which is higher than the correspond577 ing value in a grounded crystal, $c_{\mathrm{S}_{0}}=3020 \mathrm{~m} \mathrm{~s}^{-1}$. For 578 the case of $L=30 \mu \mathrm{H}$ [Fig. 8(b)], the $L$-band with ${ }_{579} c_{\text {eff }}=7400 \mathrm{~m} \mathrm{~s}^{-1}$ interacts weakly with the $A_{1}$-like 580 branch as well as with the first folded $A_{0}$-like branch, ${ }_{581}$ giving rise to relatively larger avoided crossings, occur${ }_{582} \mathrm{ring}$, respectively, at about $0.50 \mathrm{MHz}$ and $0.95 \mathrm{MHz}$; ${ }_{583}$ the other linear, $S_{0}$-like, branch has $c_{\text {eff }}=2970 \mathrm{~m} \mathrm{~s}^{-1}$, ${ }_{584}$ which is lower than $c_{\mathrm{S}_{0}}$. The above suggest a possible ${ }_{585}$ interaction with the $S_{0}$-like branch when the two modes 586 are close enough. In Fig. 8(c) we plot the evolution of ${ }_{587}$ the slope at the long-wavelength limit, for several val588 ues of the inductance covering a large range spanning 589 over two orders of magnitude, of both linear branches 590 of a typical dispersion plot as those shown in Fig. 8(a) 591 and (b). One clearly observes a hybridization between 592 two distinct modes: the $S_{0}$-like mode, represented by the 593 horizontal dashed line, and the $L$-induced mode, repre594 sented by the oblique dashed line that corresponds to 595 the $\sim L^{-1 / 2}$ scaling rule calculated at $L=10 \mu \mathrm{H}$ (for $\left.{ }_{596} C /\left[1+C /\left(4 C_{s}\right)\right]=0.618 \mathrm{nF}\right)$, far away from the crossing ${ }_{597}$ point that occurs in the vicinity of $L=150 \mu \mathrm{H}$. For this ${ }_{598}$ value of $L$, a high-degree hybridization takes place, both 599 linear-dispersion modes carry both the $S_{0}$ and $L$-induced ${ }_{600}$ character, the point A corresponding to the $L$-induced 601 band. On the contrary, for $L=30 \mu \mathrm{H}$ point $\mathrm{B}$, that 602 corresponds to the $L$-induced band, conserves mainly its ${ }_{603} L$-induced character.

604 We turn now our attention to the comparison with 605 experimental results. As previously, the $Z_{e}$ load will 606 be considered instead of the idealized $Z_{L}$ in the cal07 culations. We measured the potential signal along the $608 n=2,3, \ldots, 48$ electrode positions located at the upsog per side of the plate, and after application of the AR2D 10 model, we obtain the experimental $V\left(\omega, k_{1}\right)$ whose am11 plitude is shown in Fig. 9(a) that compares well with the 2 calculated frequency band structure including the real load $Z_{e}$, in Fig. 9(b).

The linear-slope, effective-medium branch of EM ori615 gin, clearly observed in the experiments, is perfectly re- 


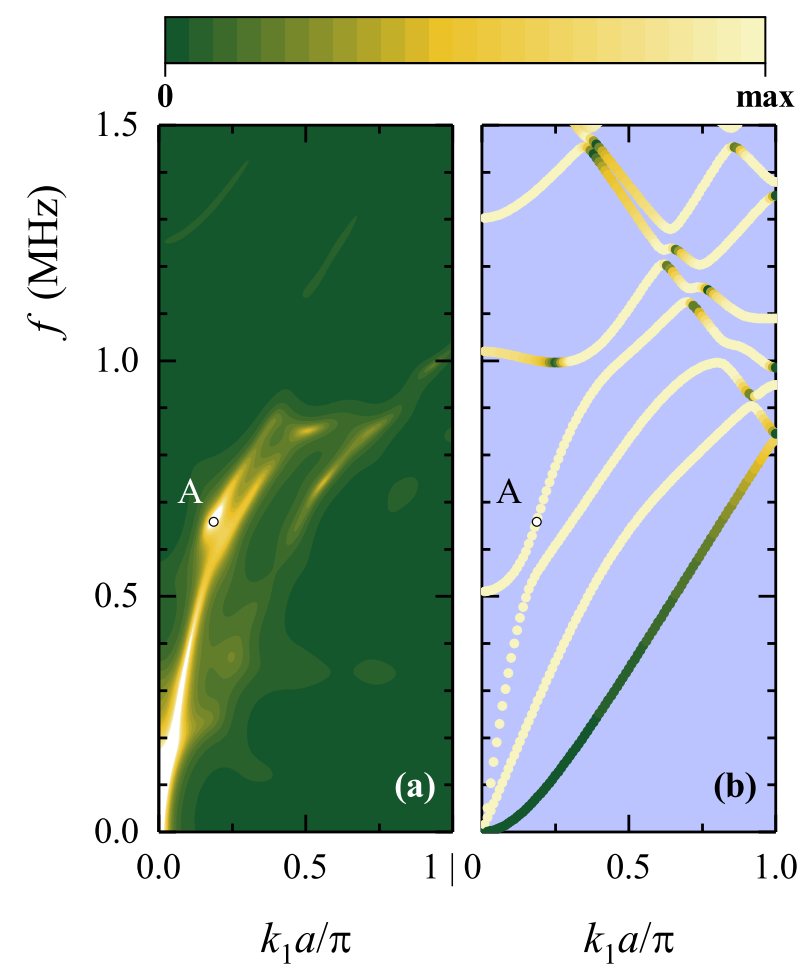

FIG. 9. (a) Experimental and (b) calculated frequency band structure for the monoatomic crystal of lattice constant $a=a_{0}$ whose unit cell is depicted in Fig. 3(c), loaded with impedance $Z_{e}$ [Eq. (B1)]. In (b) the dispersion lines are colorindexed with the projection of the potential value averaged in the upper-side electrode of the unit cell.

${ }_{616}$ produced in the calculations. The same holds for the ${ }_{617}$ bright part of the $A_{1}$-like branch [see point A in Fig. 9(a)], ${ }_{618}$ though the latter is not discernible experimentally close 619 to its cut-off frequency at $\sim 0.5 \mathrm{MHz}$ (this was also 620 the case for the crystals presented in Sec. III A and ${ }_{621}$ III B). The $S_{0}$-like branch is also observed experimen622 tally, though with lower amplitude than predicted in the ${ }_{623}$ theoretical calculation. We note that the latter fails to 624 capture the degree of repulsion of the trajectories in the ${ }_{625}$ vicinity of point $\mathrm{A}$ and for frequencies above it (this ${ }_{626}$ avoiding is predicted to be larger than experimentally ${ }_{627}$ observed). We close this part by a general remark con${ }_{628}$ cerning the $A_{0}$-like branch which is not observed (apart ${ }_{629}$ some slightly visible parts close to $\mathrm{X}$ point), since the po630 tential takes very low values along it, in all cases studied 631 here, in perfect agreement with our numerical calcula632 tions.

\section{${ }_{633}$ IV. CONCLUSIONS}

634

${ }_{635}$ In conclusion, we have presented a thorough analy${ }_{636}$ sis of the dispersion properties of piezoelectric phononic ${ }_{637}$ plates, structured with metallic arrays on their surfaces
${ }_{638}$ and loaded with inductive circuits. These structures, 639 studied experimentally and theoretically, exhibit a va640 riety of localized and/or extended in frequency modes ${ }_{641}$ that originate from an electric circuit behavior related to ${ }_{642}$ the inductive loads and span the low, intermediate, or ${ }_{643}$ high frequency range in a typical dispersion plot. This 644 low-pass, band-pass, and high-pass behavior that mani645 fests itself as an important potential variation along these 646 trajectories, combined to the automatized, controllable ${ }_{647}$ character of our experimental device, constitute a power${ }_{648}$ ful tool for applications targeting real-time manipulation 649 of elastic waves via EM waves and vice-versa. We demon650 strate the appearance of unusual, high-valued, positive or ${ }_{651}$ negative group-velocity branches, not encountered in typ652 ical Lamb-like dispersion plots of phononic plates, easily 653 tuned via the external inductive loads.

${ }_{654}$ Our experimental results, enhanced by non-ordinary, 655 high-resolution signal processing techniques, are in ac${ }_{656}$ cordance with the theoretical predictions - despite some 657 isolated discrepancies; they confirm the basic underlying 658 mechanisms analyzed in this paper and the main phe659 nomonogical aspects that interest us for applications, our 660 aim being among others the realization of viable, simple ${ }_{661}$ devices that can operate under real conditions and pro${ }_{662}$ duce the desired effects in the modulation of the disper-

${ }_{663}$ sion properties of these crystals. Nevertheless, although 664 its very application-oriented aspect at a first sight, this ${ }_{665}$ study councils per se an important physical insight.

\section{${ }_{666}$ DATA AVAILABILITY STATEMENT}

667 The data that support the findings of this study are ${ }_{668}$ available from the corresponding author upon reasonable 669 request.

\section{Appendix A: Equivalent electric circuits}

A piezoceramic plate with metallized surfaces can be 672 effectively described by an equivalent capacitor. When 673 metallic strips are structured periodically on its both sur674 faces, we can use an equivalent picture of a periodic trans675 mission line whose unit cell coincides with the unit cell of 676 the real structure. We give in what follows the electric677 circuit models that correspond to such a description for 678 the cases studied in this paper.

\section{1. Two-atom grounded- $L$ crystal}

${ }_{680}$ The equivalent circuit that models the two electric res${ }_{681}$ onators shown in Fig. 3(a) when the piezoelectric cou${ }_{682}$ pling is switched off is depicted in Fig. 10(a). We define ${ }_{683}$ all voltages in the input, $V_{A_{j}}$, and output, $V_{B_{j}}=\mathcal{P} V_{A_{j}}$,

684 of the unit cell with respect to a common ground refer${ }_{685}$ ence, where $j=1,2$, and $\mathcal{P}=\mathrm{e}^{-\mathrm{i} k_{1} a}$ is the Bloch phase ${ }_{686}$ factor (we assume an $\mathrm{e}^{+\mathrm{i} \omega t}$ time dependance in all fields); 
(a)

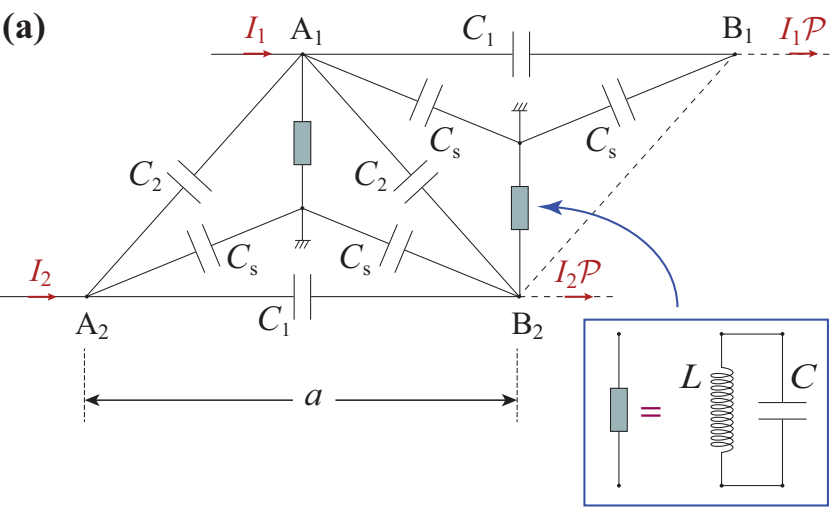

(b)

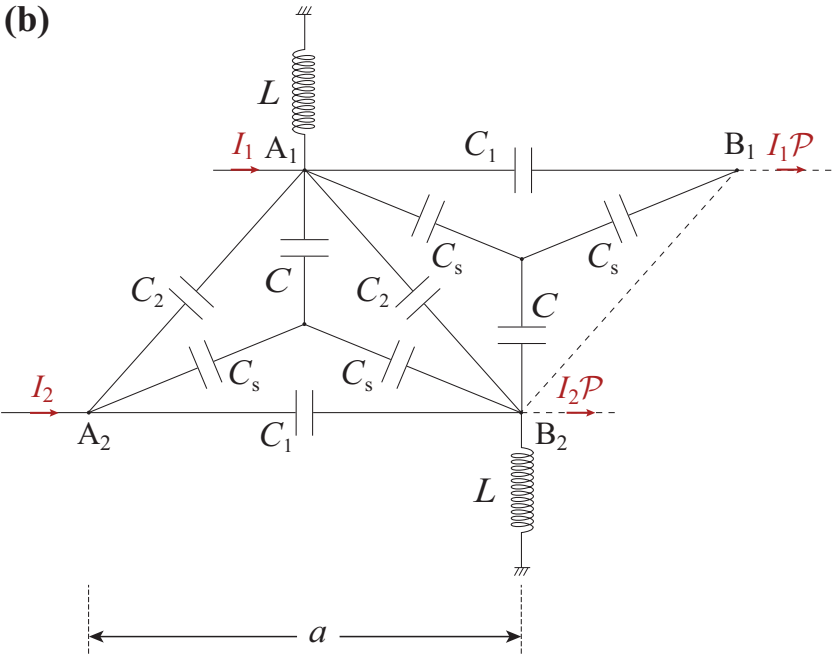

(c)

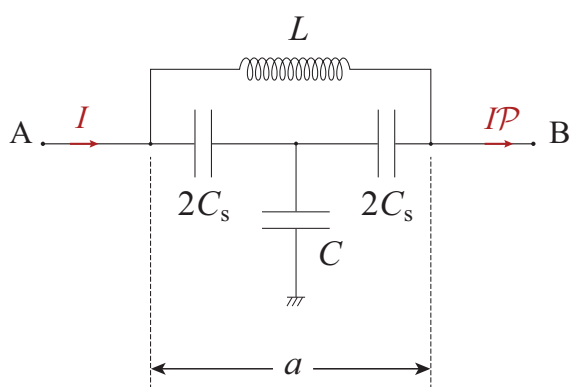

FIG. 10. The unit cell of a periodic transmission line describing equivalently (a) the two-atom grounded- $L$ crystal [Fig. 3(a)], (b) the two-atom floating-potential- $L$ crystal [Fig. 3(b)], and (c) the intercellular- $L$ crystal [Fig. 3(c)], when electromechanical coupling is switched off.

${ }_{687}$ the incoming and outgoing electric currents are respec${ }_{688}$ tively $I_{j}$ and $\mathcal{P} I_{j}$. Application of Kirchhoff's current and 689 voltage laws leads to a secular equation depending only ${ }_{690}$ on $\mathcal{P}$ and the set of unit-cell impedances. We thus ob${ }_{691}$ tain the following dispersion equation for the two electric 692 resonant bands

$$
\omega^{ \pm}\left(k_{1}\right)=\left[L C \left(1+2 \frac{C_{s}+C_{1}+C_{2}}{C}\right.\right.
$$

$$
\left.\left.-2 \frac{C_{1}}{C} \cos k_{1} a \mp 2 \frac{C_{2}}{C} \cos \frac{k_{1} a}{2}\right)\right]^{-1 / 2} .
$$

${ }_{693}$ At $\Gamma$ point (center of the BZ) the two distinct eigen${ }_{694}$ frequencies are $\omega_{0}^{+}=\left[L C\left(1+2 \frac{C_{s}}{C}\right)\right]^{-1 / 2}$ and $\omega_{0}^{-}=$ ${ }_{695}\left[L C\left(1+2 \frac{C_{s}+2 C_{2}}{C}\right)\right]^{-1 / 2}$, corresponding to a bonding and 696 an antibonding mode; at X point (edges of the BZ) a ${ }_{697}$ double degenerate eigenfrequency is found to be $\omega_{\pi}=$ ${ }_{698}\left[L C\left(1+2 \frac{C_{s}+2 C_{1}+C_{2}}{C}\right)\right]^{-1 / 2}$. The width $\Delta \omega^{ \pm}=\mid \omega_{0}^{ \pm}-$ $699 \omega_{\pi} \mid$ of these cos-like resonant bands is finite, and con700 trolled by $L$ and the internal effective parameters of the 701 model $C, C_{s}, C_{1}$ and $C_{2}$. We note that these capacitors 702 do not correspond to real electronic elements in our de703 vice, but they describe its equivalent behavior. $C$ and $C_{s}$ 704 account for a capacitive effect between electrodes located 705 at the two opposite sides (i.e., along $x_{3}$-direction) and at 706 the same side (i.e., along $x_{1}$-direction) of the plate, re707 spectively. $C_{1}$ and $C_{2}$ describe the capacitive interaction 708 between electrodes of the same side at distance $a=2 a_{0}$ 709 and between electrodes at the opposite sides at distance ${ }_{710} R=\sqrt{a_{0}^{2}+h^{2}}$, respectively, in one-to-one analogy with 711 the overlapping coefficients $\gamma_{1}, \gamma_{2}$ of the tight-binding 712 model.

\section{2. Two-atom floating-potential- $L$ crystal}

${ }_{714}$ For the case of the two-atom crystal with an alterna715 tion of floating-potential EBCs and inductance loads 716 its unit cell is given in Fig. 3(b) - we proceed in a similar 717 manner to construct its equivalent circuit [see Fig. 10(b)] 718 that models the two electric resonators. Using the same 719 steps as for the previous case, we obtain, after a rela720 tively lengthy but straightforward algebra, the following 721 dispersion equation for the two electric bands

$$
\begin{aligned}
\omega^{ \pm}\left(k_{1}\right) & =\left\{2 L C \left[\frac{C_{1}+2 C_{2}}{C}+\frac{C_{s}}{C} \frac{2 C+C_{s}}{C+2 C_{s}}\right.\right. \\
& -\left(\frac{C_{1}}{C}+\frac{C_{s}}{C} \frac{C_{s}}{C+2 C_{s}}\right) \cos k_{1} a \\
& \left.\left.\mp 2\left(\frac{C_{2}}{C}+\frac{C_{s}}{C+2 C_{s}}\right) \cos \frac{k_{1} a}{2}\right]\right\}^{-1 / 2} .
\end{aligned}
$$

${ }_{722}$ At $\Gamma$ point two distinct eigensolutions are found, one fi${ }_{723}$ nite $\omega_{0}^{-}=\left[8 L C_{2}\left(1+\frac{C}{C_{2}} \frac{C_{s}}{2 C_{s}+C}\right)\right]^{-1 / 2}$ and one diverg${ }_{724}$ ing as $\omega_{0}^{+} \sim\left[L C_{1}\left(1+\frac{C_{s}}{C_{1}} \frac{C_{s}}{2 C_{s}+C}\right)\right]^{-1 / 2}\left(k_{1} a\right)^{-1}$. The 725 higher frequency branch $\omega^{+}\left(k_{1}\right)$ behaves as an hyper726 bolic function for $k_{1} a \ll 1$, while the lower frequency 727 branch $\omega^{-}\left(k_{1}\right)$ corresponds to a cos-like resonant band 728 of finite width $\Delta \omega^{-}=\left|\omega_{0}^{-}-\omega_{\pi}\right|$, where $\omega_{\pi}$ is the com729 mon, double degenerate eigenfrequency at $\mathrm{X}$ point, found 730 to be $\omega_{\pi}=\left[4 L\left(C_{1}+C_{2}+C_{s} \frac{C+C_{s}}{C+2 C_{s}}\right)\right]^{-1 / 2}$. The form 731 of these two dispersive modes is still controlled by $L$ and ${ }_{732}$ the internal effective parameters of the model $C, C_{s}, C_{1}$ 

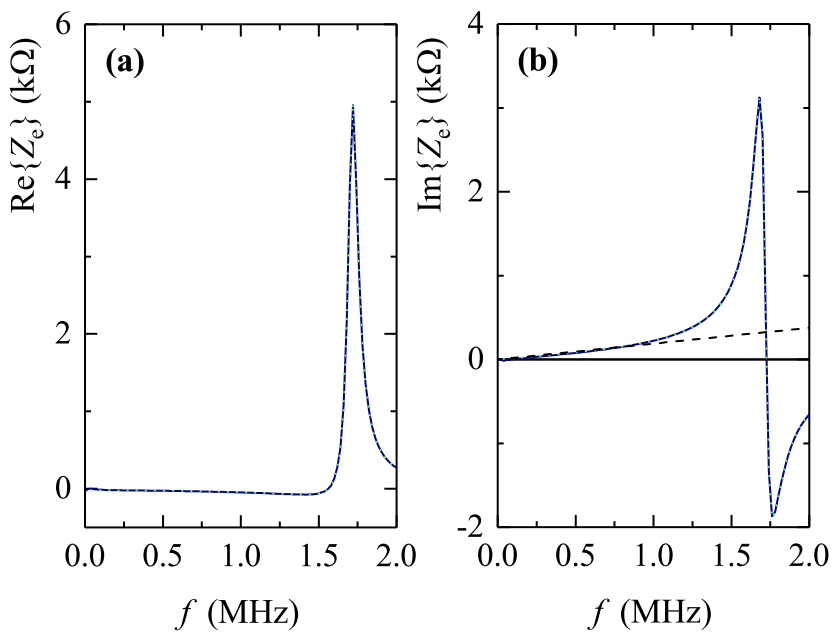

FIG. 11. Real (a) and imaginary (b) part of the impedance load $Z_{e}$ used in the external circuits of Fig. 3. Solid and dotted lines represent the real (measured) and modeled [Eq. (B1)] function, respectively; in (b) the impedance of a pure inductance $L=30 \mu \mathrm{H}$ is also shown (broken line) for comparison.

733 and $C_{2}$, whose physical meaning is the same as that for 734 the grounded- $L$ crysral.

\section{3. Intercellular- $L$ crystal}

${ }_{736}$ The unit cell of Fig. 3(c) is modeled by the periodic 737 transmission line whose unit cell is shown in Fig. 10(c). ${ }_{738}$ We easily obtain the following dispersion relation

$$
\begin{aligned}
\omega\left(k_{1}\right) & =\omega_{0}\left[\frac{C}{4 C_{s}}+\left(2 \sin \frac{k_{1} a}{2}\right)^{-2}\right]^{-1 / 2}, \\
\omega_{0} & =\left[\frac{1}{L C}\left(1+\frac{C}{4 C_{s}}\right)\right]^{1 / 2}
\end{aligned}
$$

739 from which one can immediately deduce the angular fre740 quency at $\mathrm{X}$ point, $\omega_{\pi}=\frac{1}{\sqrt{L C_{s}}}$, and the effective medium 76 ${ }_{741}$ velocity at the long wavelength limit $c_{\text {eff }}=\lim _{k_{1} \rightarrow 0} \frac{\omega}{k_{1}}={ }^{767}$ ${ }_{742} a \omega_{0}$. In this simplified but still quite accurate model, ${ }_{769}^{768}$ 743 knowledge of two external parameters, e.g. $\omega_{\pi}$ and $\omega_{0} 770$ 744 (or, equivalently, $c_{\mathrm{eff}}$ ), is sufficient to determine the in- 771 745 ternal model parameters, $C$ and $C_{s}$.

\section{Appendix B: Impedance-load function}

${ }_{747}$ The external impedance load is ideally assumed to be 748 an inductance $L$ connected to the metallic strips, as de749 tailed in Fig. 3. However, in practice, the real compo- 78 750 nent, used in our experiments, has not a pure inductive ${ }^{782}$ 751 behavior; other contributions have to be taken into ac- ${ }^{783}$ ${ }_{752}$ count, related to several internal circuitry parts of re- ${ }^{784}$ 753 sistive and/or capacitive type of this component. The ${ }_{786}^{785}$ ${ }_{754}$ measured impedance $Z_{\mathrm{e}}$ of our individual component $-\mathrm{a} \quad{ }_{787}$
755 representative one, extracted from the crystal- is plot756 ted in Fig. 11 against frequency, $f$, and both its real and 757 imaginary parts [plots (a) and (b), respectively] show a 758 resonant behavior at about $1.71 \mathrm{MHz}$. Within the fre759 quency range that interest us here (up to $2 \mathrm{MHz}$ ) this 760 function can be in a very good approximation described 761 by the following rational expression

$$
\begin{aligned}
Z_{\mathrm{e}} & =\frac{\alpha_{4} f^{4}+\alpha_{3} f^{3}+\alpha_{2} f^{2}+\alpha_{1} f+\alpha_{0}}{f^{2}+\beta_{1} f+\beta_{0}} \\
& =\gamma_{0}+\gamma_{1} f+\gamma_{2} f^{2}+\frac{\delta_{+}}{f-f_{+}}+\frac{\delta_{-}}{f-f_{-}}
\end{aligned}
$$

762 where $f$ is expressed in $\mathrm{MHz}$ in the above expressions, 763 and $\alpha_{n}$ and $\beta_{n}$ are appropriate complex coefficients ob764 tained by a fitting procedure

$$
\begin{aligned}
& \alpha_{4}=-5.15566-\mathrm{i} 5.18013 \times 10^{-14}, \\
& \alpha_{3}=-6.96706 \times 10^{-14}+\mathrm{i} 22.0970, \\
& \alpha_{2}=52.4644+\mathrm{i} 4.78721 \times 10^{-13}, \\
& \alpha_{1}=4.56112 \times 10^{-13}-\mathrm{i} 452.779, \\
& \alpha_{0}=56.4767-\mathrm{i} 7.41831 \times 10^{-13}, \\
& \beta_{1}=-7.61040 \times 10^{-17}-\mathrm{i} 0.0790166, \\
& \beta_{0}=-2.94215+\mathrm{i} 8.96296 \times 10^{-18},
\end{aligned}
$$

765 and

$$
\begin{aligned}
\gamma_{0} & =\alpha_{2}-\alpha_{3} \beta_{1}+\alpha_{4}\left(\beta_{1}^{2}-\beta_{0}\right) \\
& =35.5818+\mathrm{i} 3.22798 \times 10^{-13} \\
\gamma_{1} & =\alpha_{3}-\alpha_{4} \beta_{1} \\
& =-6.59699 \times 10^{-14}+\mathrm{i} 21.6896 \\
\gamma_{2} & =\alpha_{4} \\
\delta_{ \pm} & = \pm 51.4400-\mathrm{i} 193.077 \\
f_{ \pm} & =\frac{1}{2}\left(-\beta_{1} \pm \sqrt{\beta_{1}^{2}-4 \beta_{0}}\right) \\
& = \pm 1.71481+\mathrm{i} 0.0395083 .
\end{aligned}
$$

${ }^{1}$ M. Leamy, M. Carrara, A. Erturk, M. Cacan, and M. Ruzzene, "Metamaterial-inspired structures and concepts for elastoacoustic wave energy harvesting," Smart Mater. Struct. 22, 065004 (2013).

${ }^{2}$ J. Li, X. Zhou, G. Huang, and G. Hu, "Acoustic metamaterials capable of both sound insulation and energy harvesting," Smart Mater. Struct. 25, 045013 (2016).

${ }^{3} \mathrm{~L}$. Wu, Y. Wang, K. Chuang, F. Wu, Q. Wang, W. Lin, and H. Jiang, "A brief review of dynamic mechanical metamaterials for mechanical energy manipulation," Mater. Today 44, 168-193 (2021).

4Z. Liu, X. Zhang, Y. Mao, Y.Y. Zhu, Z. Yang, C.T. Chan, and P. Sheng, "Locally resonant sonic materials," Science 289, 17341736 (2000).

${ }^{5}$ J. Li and C.T. Chan, "Double-negative acoustic metamaterial," Phys. Rev. E 70, 055602(R) (2004).

${ }^{6}$ A. Ba, A. Kovalenko, C. Aristégui, O. Mondain-Monval, and T. Brunet, "Soft porous silicone rubbers with ultra-low sound speeds in acoustic metamaterials," Sci. Rep. 7, 40106 (2017).

P. Celli, B. Yousefzadeh, C. Daraio, and S. Gonella, "Bandgap widening by disorder in rainbow metamaterials," Appl. Phys. Lett. 114, 091903 (2019). 
${ }_{88}^{8}$ M. Miniaci, A. Krushynska, A.S. Gliozzi, N. Kherraz, F. Bosia, 830 and N.M. Pugno, "Design and Fabrication of Bioinspired Hier- 831 archical Dissipative Elastic Metamaterials," Phys. Rev. Applied 832 10, 024012 (2018).

$792{ }^{9}$ Q.J. Lim, P. Wang, S.J.A. Koh, E.H. Khoo, and K. Bertoldi, 834 "Wave propagation in fractal-inspired self-similar beam lattices," 835 Appl. Phys. Lett. 107, 221911 (2015).

$95{ }^{10}$ J.-F. Robillard, O. Bou Matar, J.O. Vasseur, P.A. Deymier, ${ }_{837}$ 796 M. Stippinger, A.-C. Hladky-Hennion, Y. Pennec, and B. Djafari- 838 797 Rouhani, "Tunable magnetoelastic phononic crystals," Appl. 839 98 Phys. Lett. 95, 124104 (2009).

${ }^{11}$ W. Qian, Z. Yu, X. Wang, Y. Lai, and B.B. Yellen, "Elastic 800 metamaterial beam with remotely tunable stiffness," J. Appl. 842 801 Phys. 119, 055102 (2016)

${ }_{802}{ }^{12}$ S.W. Xiao, G.C. Ma, Y. Li, Z.Y. Yang, and P. Sheng, "Ac- 844 803 tive control of membrane-type acoustic metamaterial by electric 845 804 field," Appl. Phys. Lett. 106, 91904 (2015).

${ }_{805}{ }^{13}$ Y. Cheng, X.J. Liu, and D.J. Wu, "Temperature effects on the 847 806 band gaps of lamb waves in a one-dimensional phononic-crystal 848 807 plate," J. Acoust. Soc. Am. 129, 1157-1160 (2011).

${ }_{808}^{14} \mathrm{Z}$. Li, Y. Li, S. Kumar, and H.P. Lee, "Thermal tuning of negative 850 809 effective mass density in a two-dimensional acoustic metamaterial 85 810 with hexagonal lattice," J. Appl. Phys. 126, 155102 (2019).

${ }_{811}^{15}$ E. Walker, D. Reyes, M.M. Rojas, A. Krokhin, Z. Wang, and 853 812 A. Neogi, "Tunable ultrasonic phononic crystal controlled by in- 854 813 frared radiation," Appl. Phys. Lett. 105, 143503 (2014). 855

$814{ }^{16}$ A.S. Gliozzi, M. Miniaci, A. Chiappone, A. Bergamini, B. Morin, 856 815 E. Descrovi, "Tunable photo-responsive elastic metamaterials," 857 $816 \quad$ Nat. Comm. 11, 2576 (2020).

${ }_{817}{ }^{17} \mathrm{~L}$. Airoldi and M. Ruzzene, "Design of tunable acoustic metama-

18 terials through periodic arrays of resonant shunted piezos," New J. Phys. 13, 113010 (2011).

$820{ }^{18}$ R. Zhu, Y.Y. Chen, M.V. Barnhart, G.K. Hu, C.T. Sun, and

821 G.L. Huang, "Experimental study of an adaptive elastic meta822 material controlled by electric circuits," Appl. Phys. Lett. 108, $023 \quad 011905$ (2016)

${ }^{824}{ }^{19}$ C. Sugino, M. Ruzzene, and A. Erturk, "Design and analysis 825 of piezoelectric metamaterial beams with synthetic impedance 826 shunt circuits," IEEE/ASME Trans. Mechatronics 23, 2144-55 827 (2018).

${ }_{828}{ }^{20}$ S. Degraeve, C. Granger, B. Dubus, J.O. Vasseur, M. Pham Thi, ${ }_{870}^{869}$ 29 and A.-C. Hladky-Hennion, "Bragg band gaps tunability in an 871 homogeneous piezoelectric rod with periodic electrical boundary conditions," J. Appl. Phys. 115, 194508 (2014).

${ }^{11}$ S. Degraeve, C. Granger, B. Dubus, J.O. Vasseur, M. Pham Thi and A.-C. Hladky-Hennion, "Tunability of Bragg band gaps in one-dimensional piezoelectric phononic crystals using external capacitances," Smart Mater. Struct. 24, 085013 (2015).

${ }^{22}$ S.A. Mansoura, P. Benard, B. Morvan, P. Marechal, A.C. Hladky-Hennion, and B. Dubus, "Theoretical and experimental analysis of a piezoelectric plate connected to a negative capacitance at MHz frequencies," Smart Mater. Struct. 24, 115032 (2015).

33 N. Kherraz, L. Haumesser, F. Levassort, P. Benard, and B. Morvan, "Controlling Bragg gaps induced by electric boundary conditions in phononic piezoelectric plates," Appl. Phys. Lett. 108, 093503 (2016)

${ }^{24}$ N. Kherraz, L. Haumesser, F. Levassort, P. Benard, and B. Morvan, "Hybridization bandgap induced by an electrical resonance in piezoelectric metamaterial plates," J. Appl. Phys. 123, 094901 (2018).

${ }^{25}$ N. Kherraz, F.H. Chikh-Bled, R. Sainidou, B. Morvan, and P. Rembert, "Tunable phononic structures using Lamb waves in a piezoceramic plate," Phys. Rev. B 99, 094302 (2019).

${ }^{26}$ F.H. Chikh-Bled, N. Kherraz, R. Sainidou, P. Rembert, and B. Morvan, "Piezoelectric phononic plates: retrieving the frequency band structure via all-electric experiments," Smart Mater. Struct. 28, 115046 (2019).

${ }^{27}$ We used the COMSOL Multiphysics v. 5.5 software to perform the calculations (www.comsol.com. COMSOL AB, Stockholm, Sweden).

${ }^{28}$ https : //www . americanpiezo.com/.

${ }^{29} \mathrm{M}$. Tummala, "New algorithm for solving block matrix equations with applications in 2-D AR spectral estimation," IEEE Trans. Signal Processing 39, 759-764 (1991).

${ }^{30}$ N. W. Ashcroft and N. D. Mermin, Solid State Physics (New York: Saunders College Publishing, 1976).

${ }^{31}$ The equivalent electric circuit that models this unit cell coincides to that shown in Fig.A1(b) of Ref. 26, and the dispersion relation of this mode is still given by Eq.(2) of the above-mentionned reference.

${ }^{32}$ R. Sainidou, N. Stefanou, I.E. Psarobas, and A. Modinos, "Scattering of elastic waves by a periodic monolayer of spheres," Phys. Rev. B 66, 024303 (2002). 

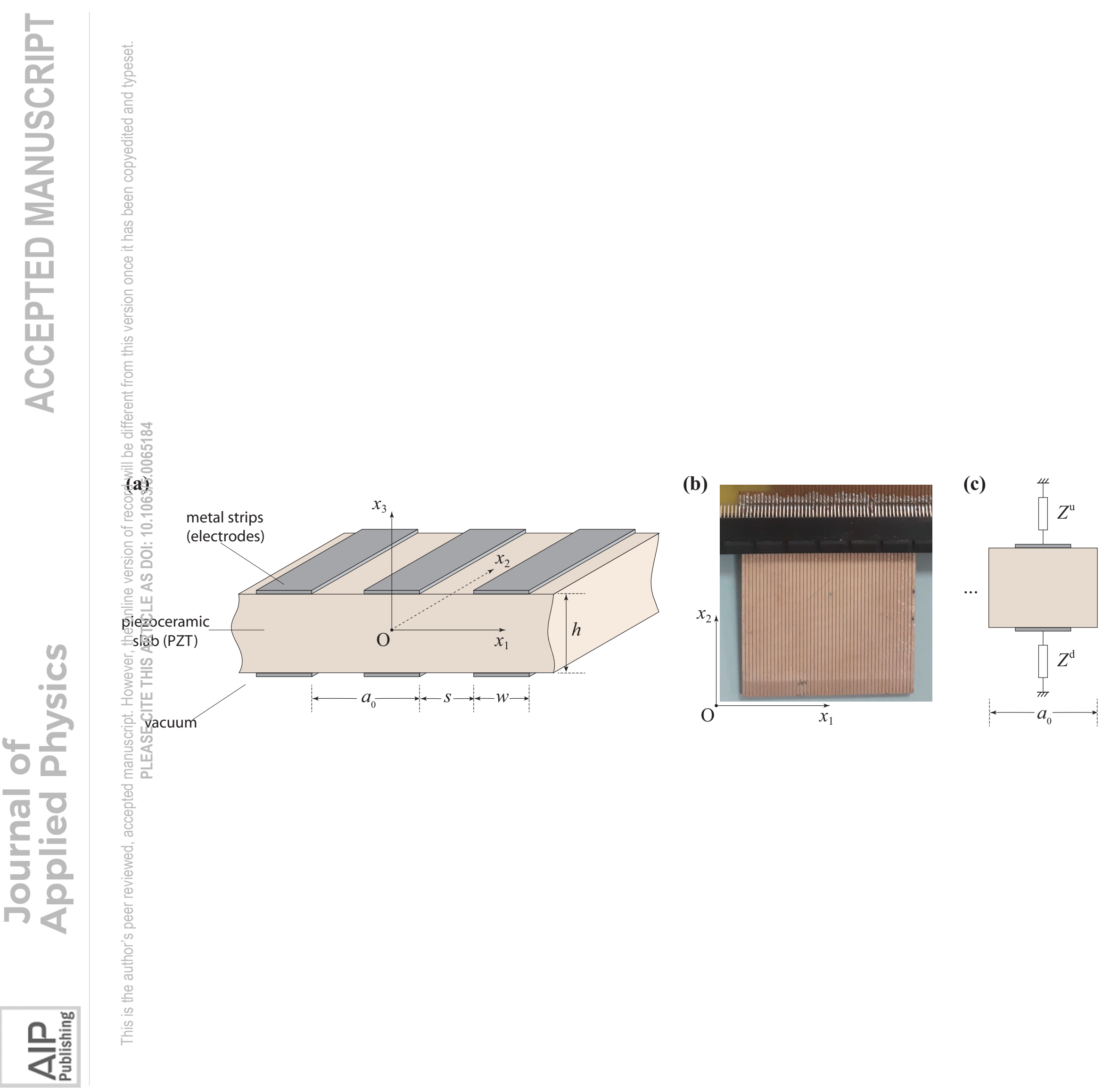
(a) $\mathrm{PCl}$ card

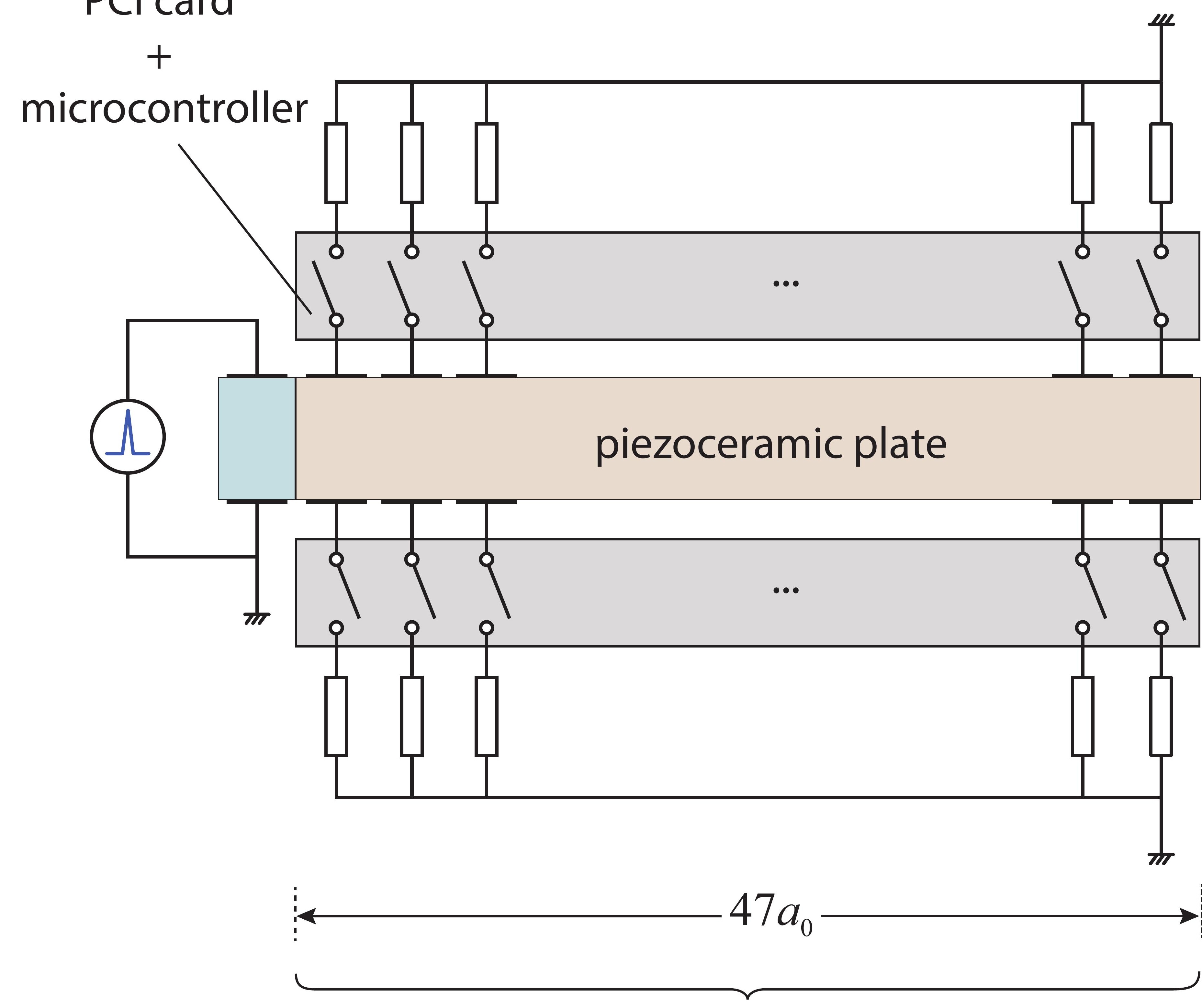

Phononic crystal with actuated loads

(b)

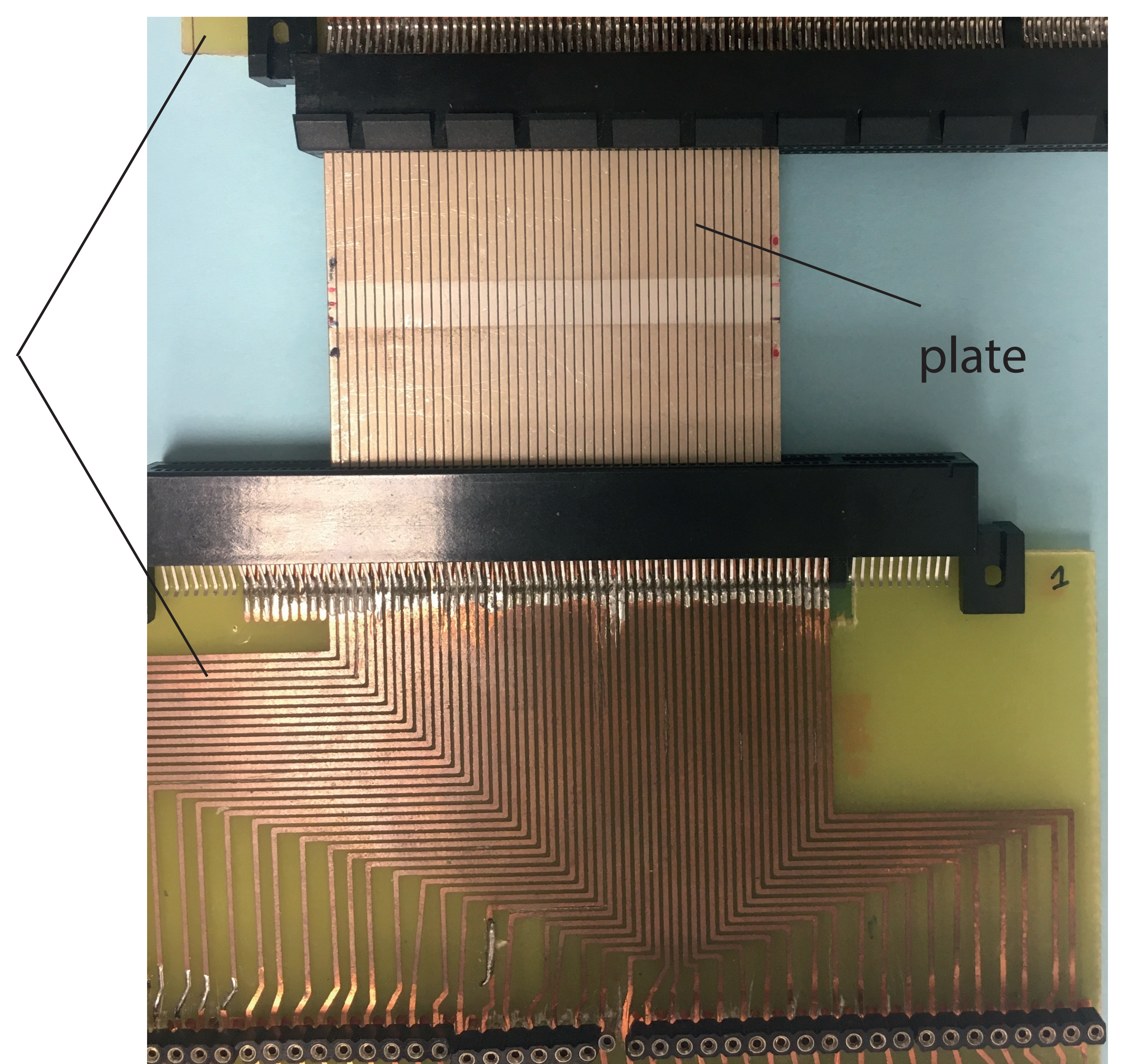


(a)
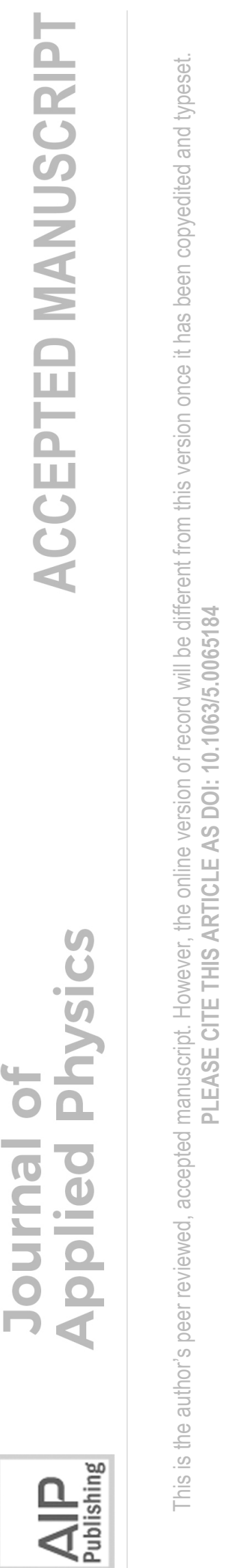

(b)

(c)
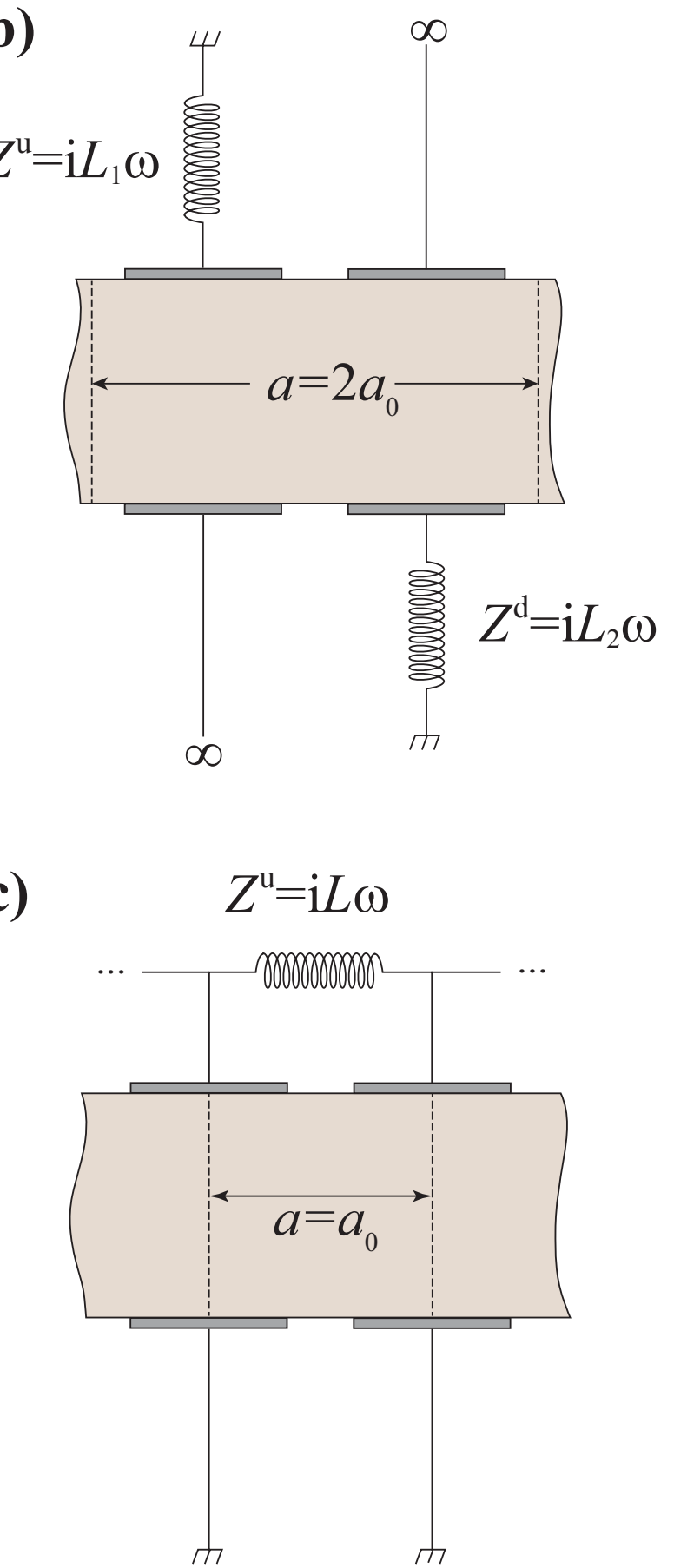
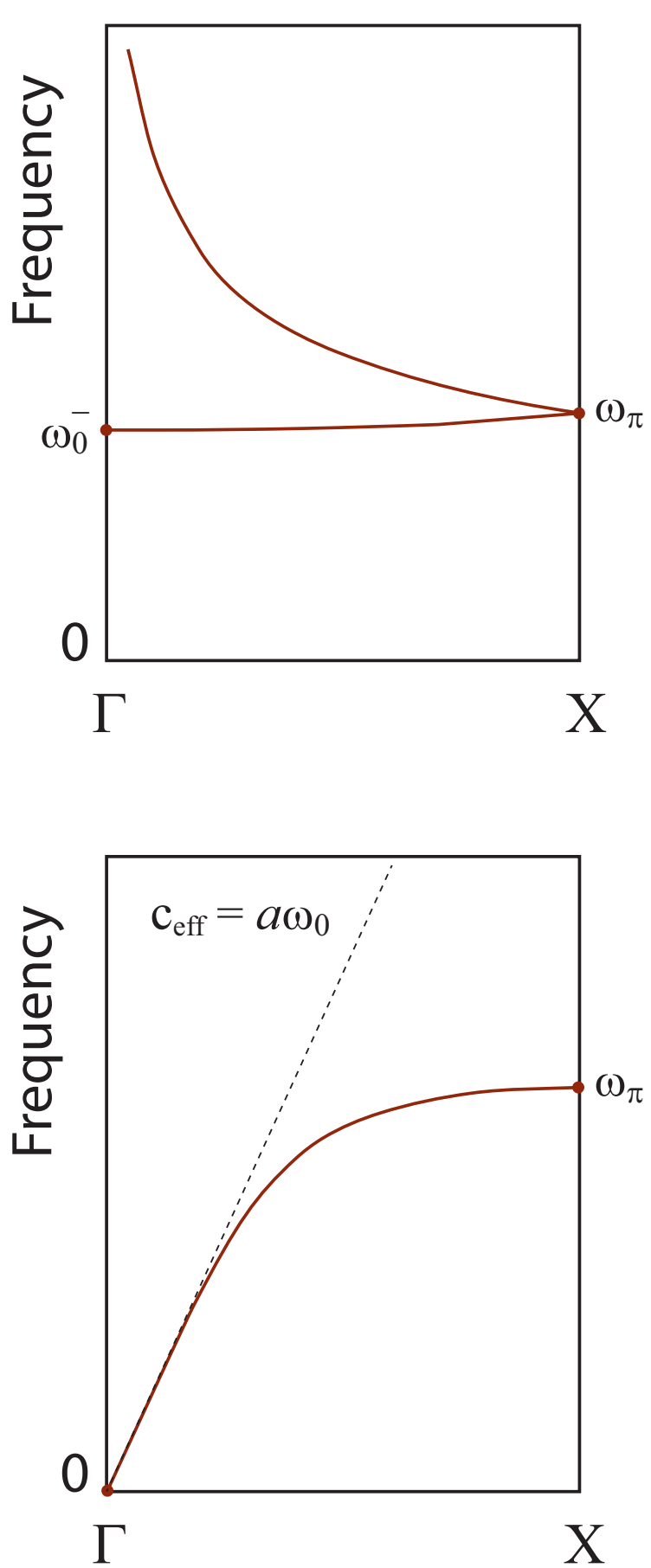


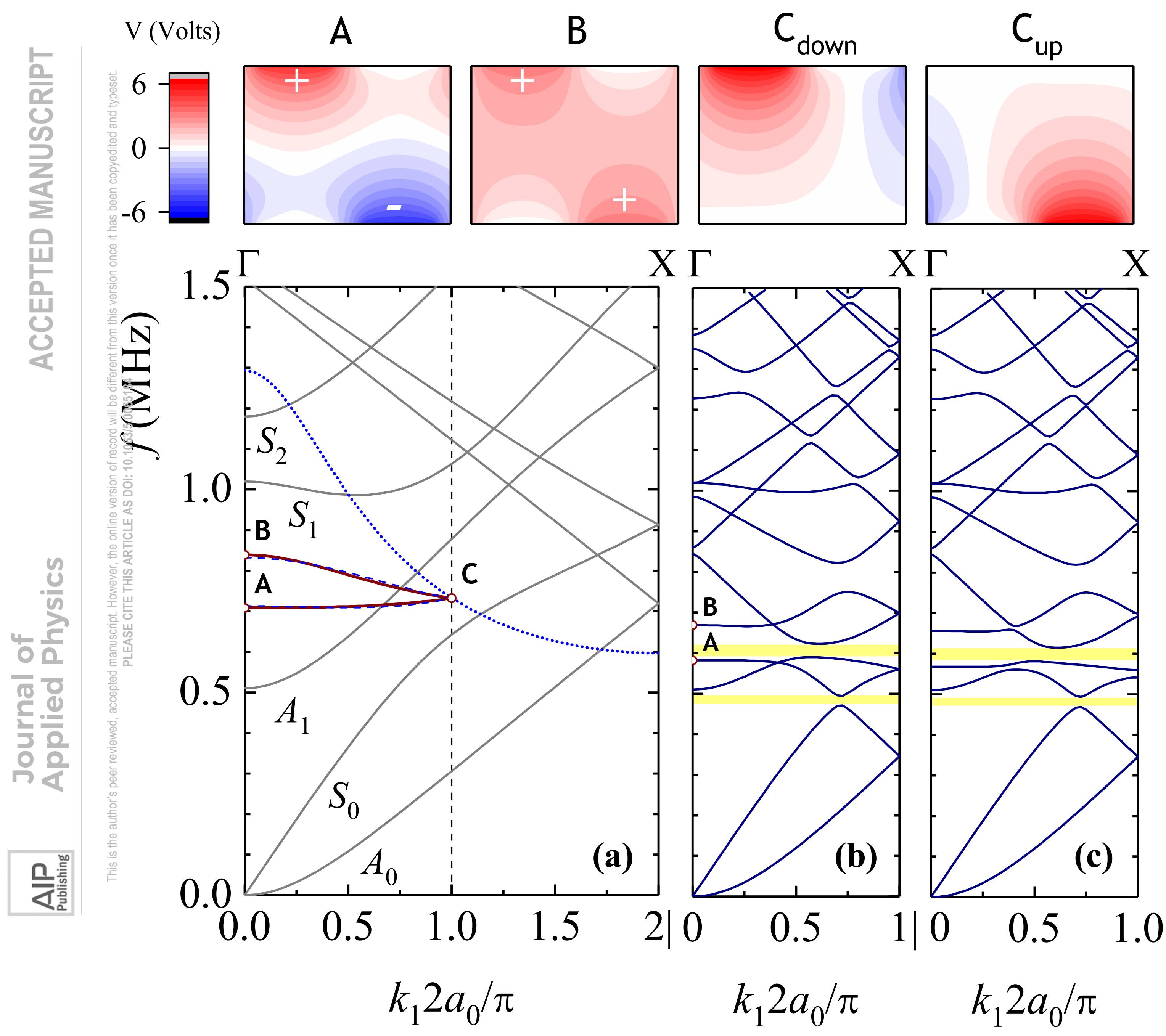




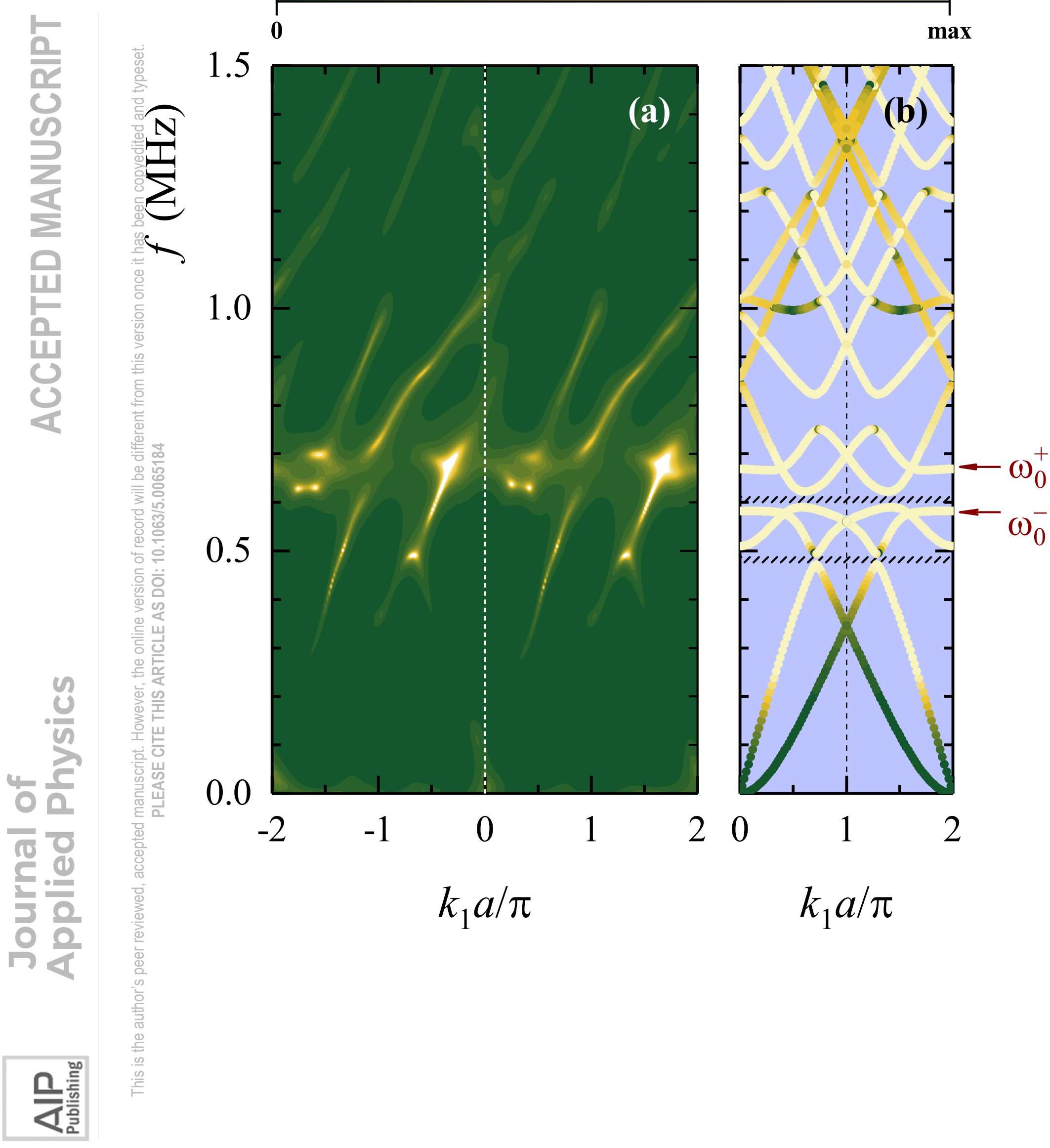




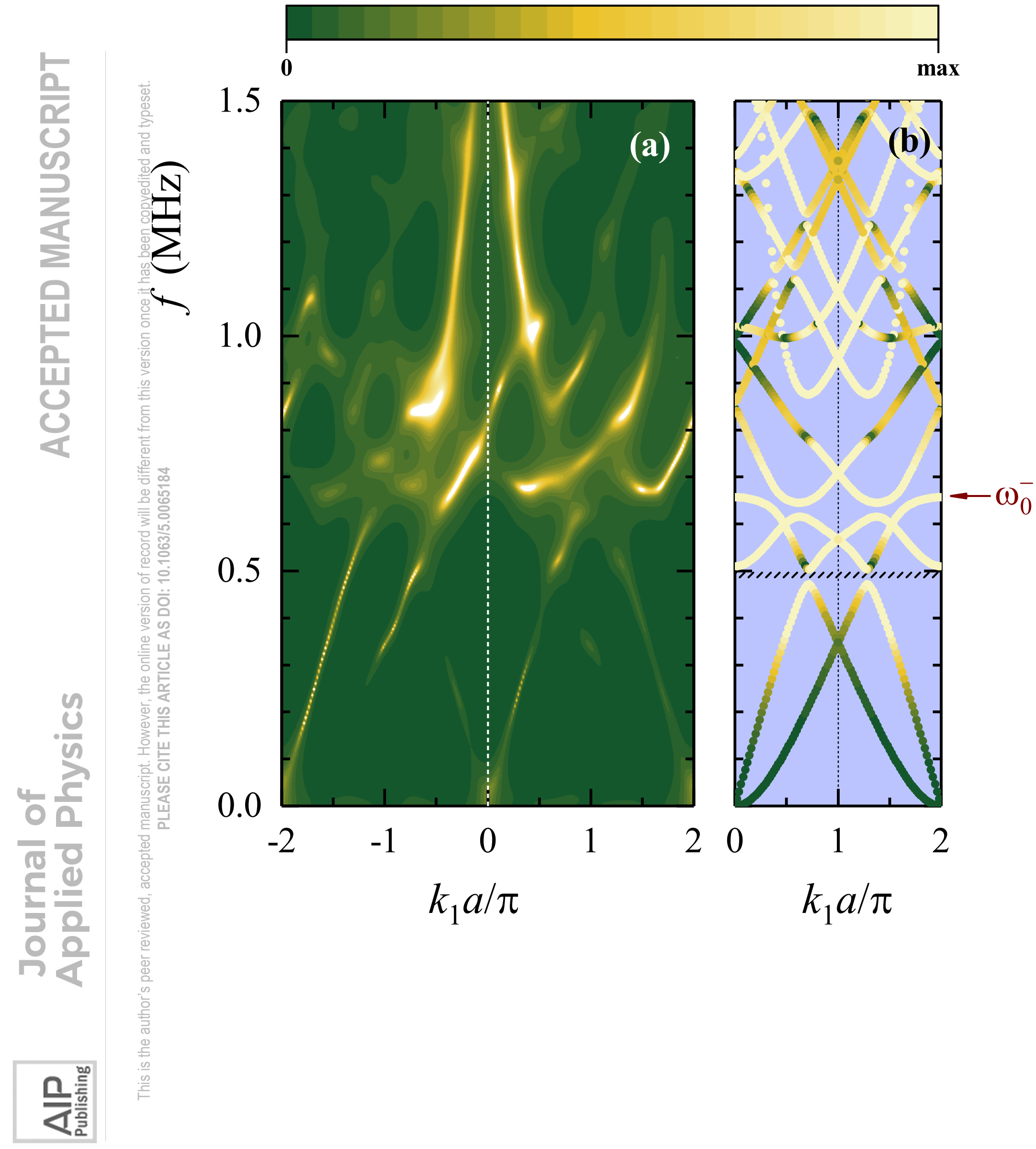




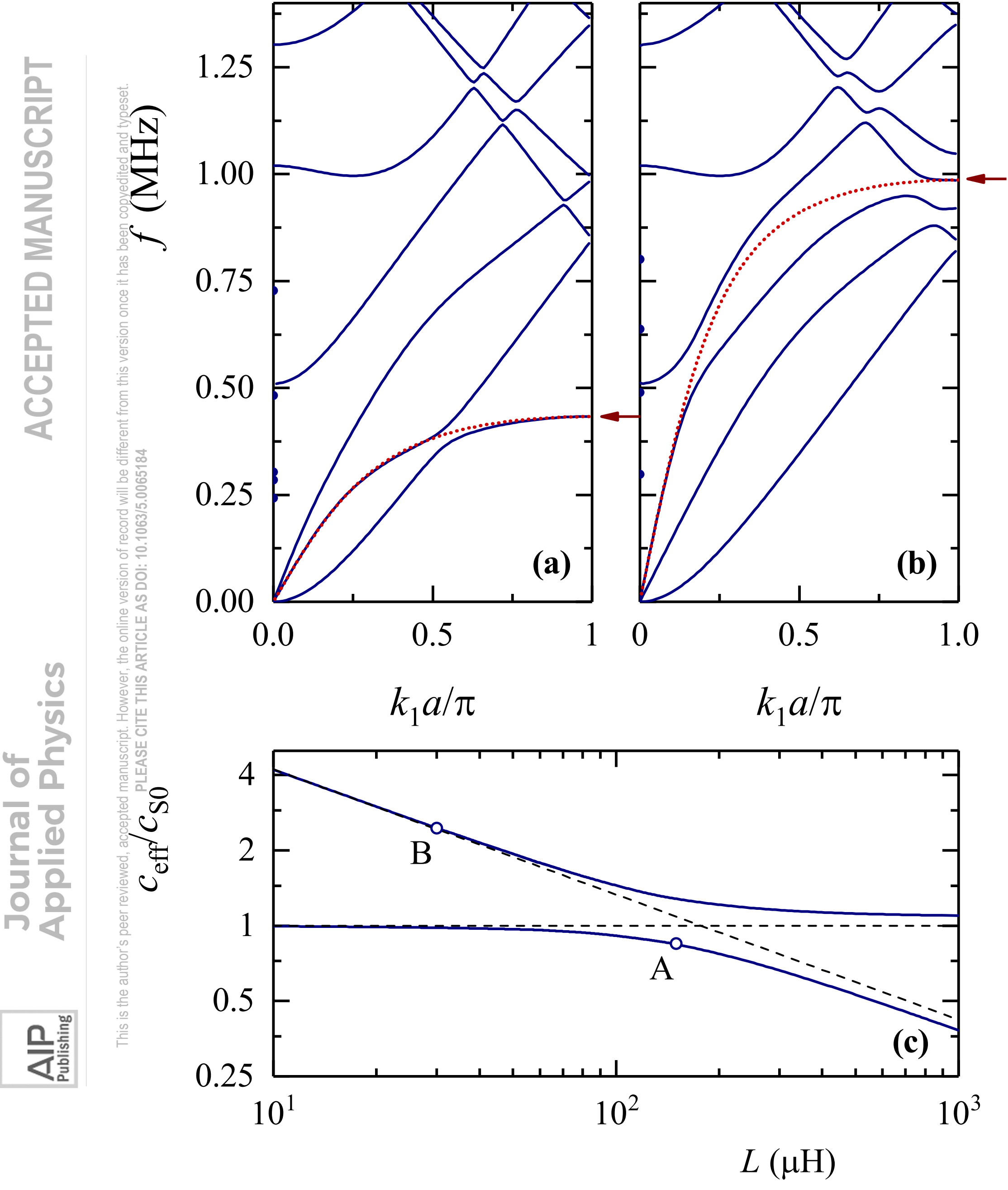




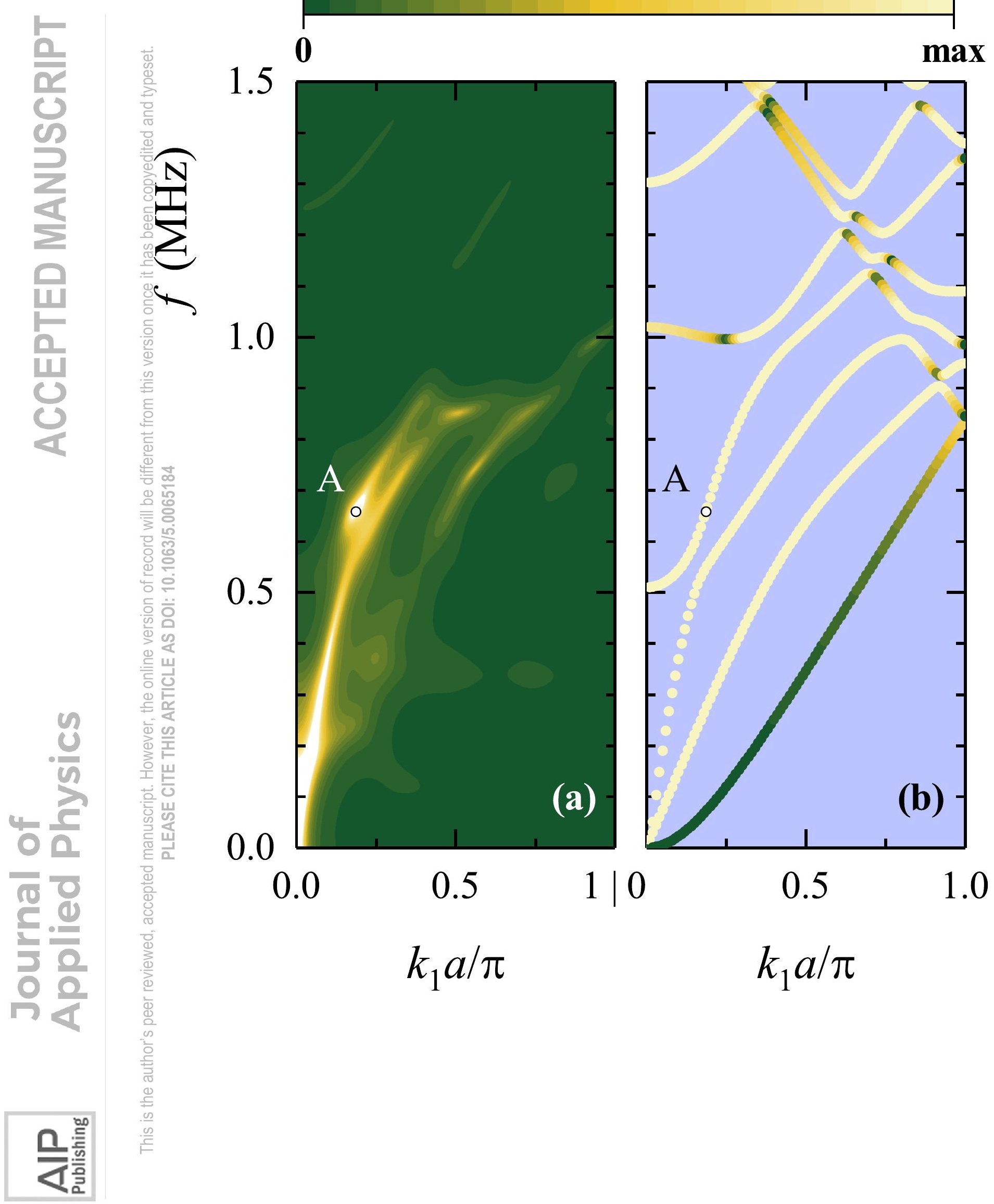


(a)

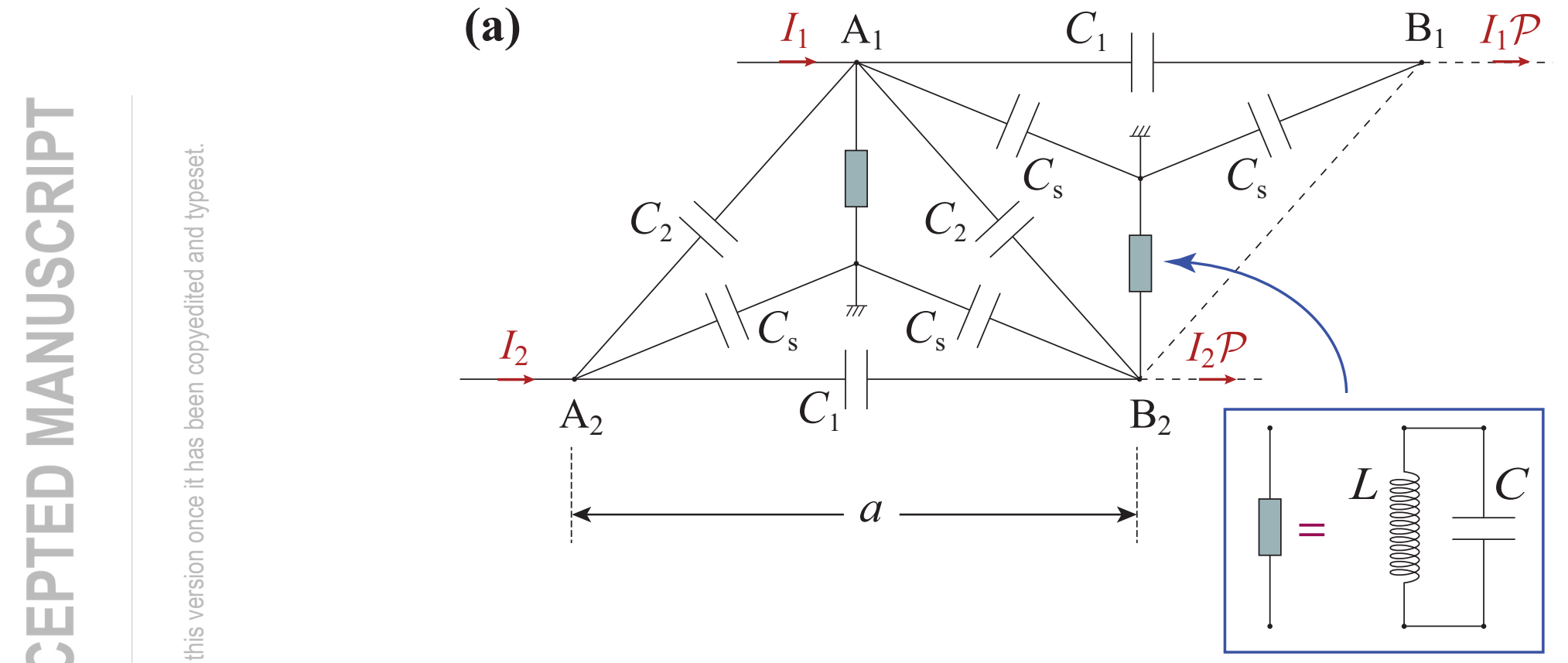

(b)

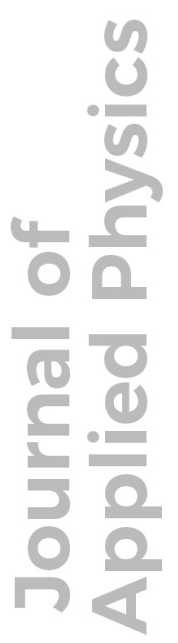
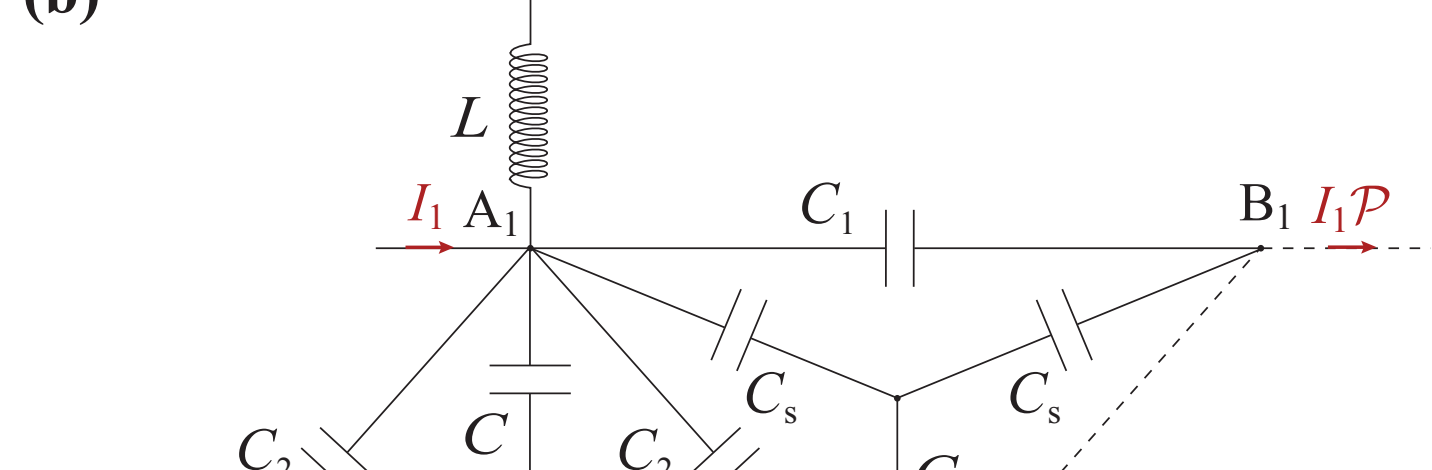

(c)

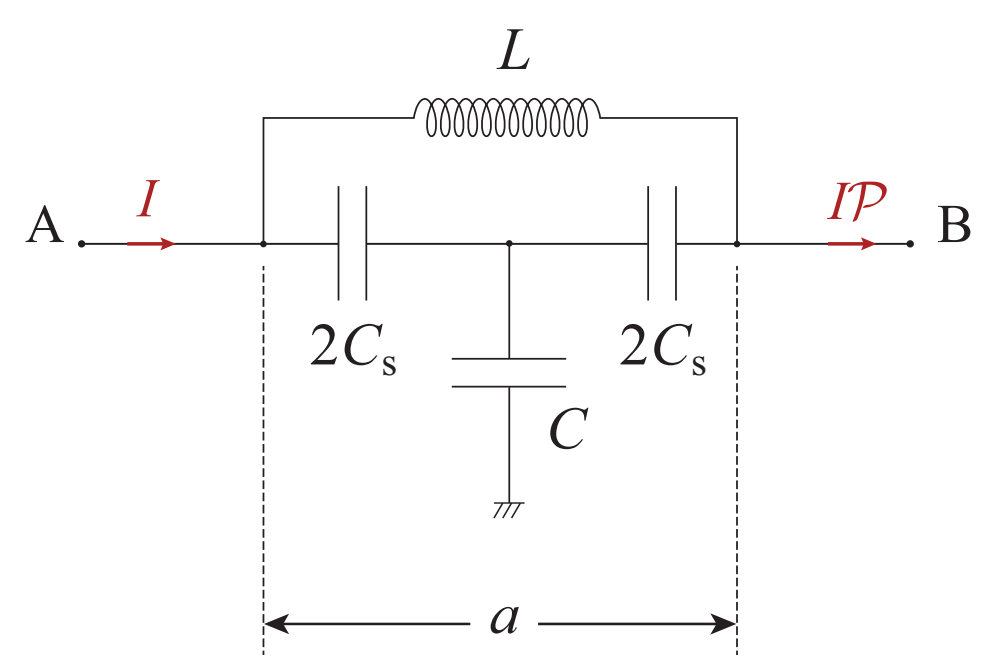




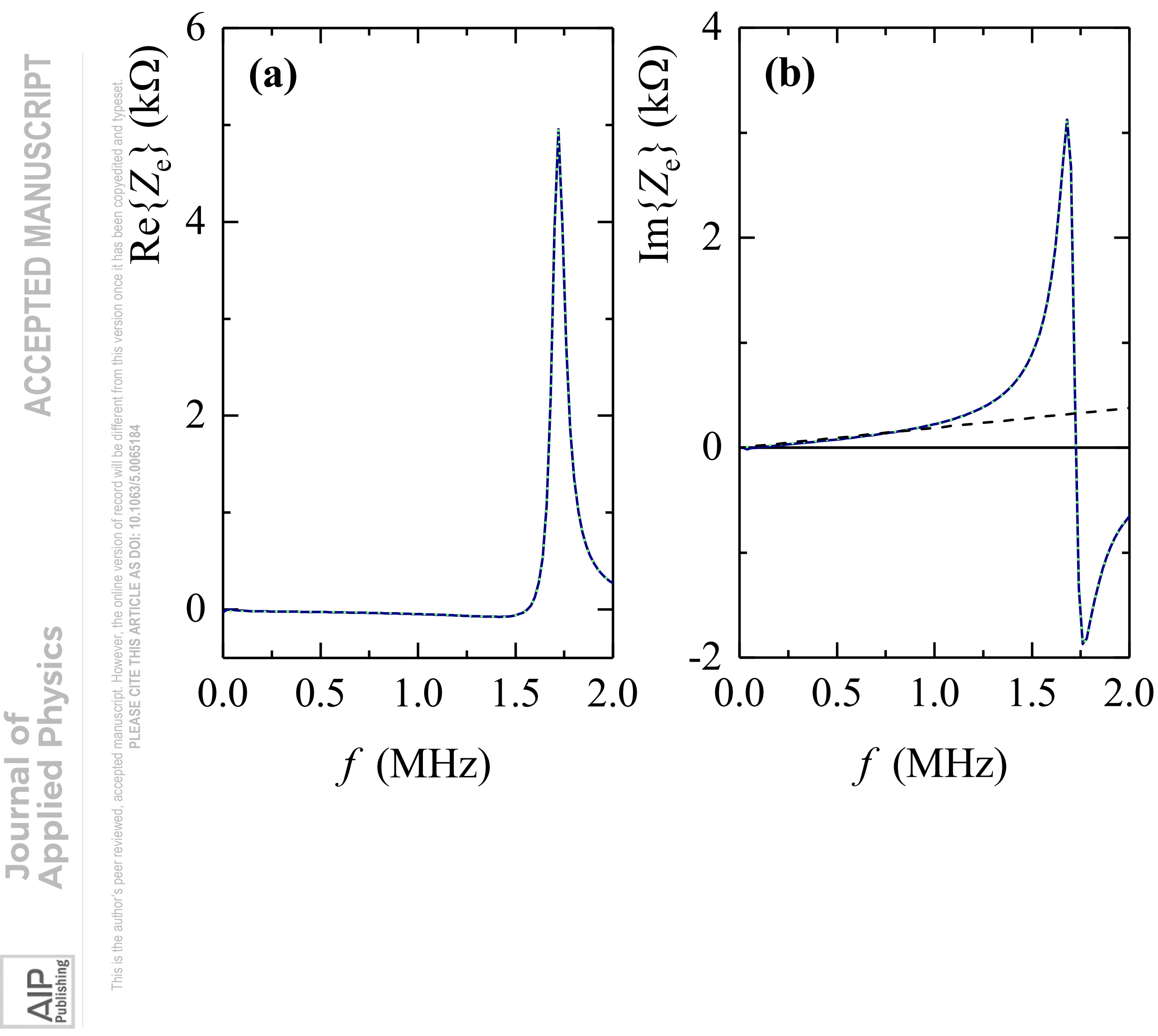

